\title{
Probing the cosmic distance-duality relation with the Sunyaev-Zel'dovich effect, X-ray observations and supernovae la
}

\author{
R. F. L. Holanda ${ }^{1}$, J. A. S. Lima ${ }^{1}$, and M. B. Ribeiro ${ }^{2}$ \\ ${ }^{1}$ Departamento de Astronomia, Instituto Astronômico e Geofísico, Universidade de São Paulo - USP, São Paulo, Brazil \\ e-mail: [holanda;limajas]@astro.iag.usp.br \\ 2 Instituto de Física, Universidade Federal do Rio de Janeiro - UFRJ, Rio de Janeiro, Brazil \\ e-mail: mbr@if.ufrj.br
}

Received 27 October 2011 / Accepted 27 November 2011

\begin{abstract}
Context. The angular diameter distances toward galaxy clusters can be determined with measurements of Sunyaev-Zel'dovich effect and X-ray surface brightness combined with the validity of the distance-duality relation, $D_{\mathrm{L}}(z)(1+z)^{2} / D_{\mathrm{A}}(z)=1$, where $D_{\mathrm{L}}(z)$ and $D_{\mathrm{A}}(z)$ are, respectively, the luminosity and angular diameter distances. This combination enables us to probe galaxy cluster physics or even to test the validity of the distance-duality relation itself.

Aims. We explore these possibilities based on two different, but complementary approaches. Firstly, in order to constrain the possible galaxy cluster morphologies, the validity of the distance-duality relation (DD relation) is assumed in the $\Lambda$ CDM framework (WMAP7). Secondly, by adopting a cosmological-model-independent test, we directly confront the angular diameters from galaxy clusters with two supernovae Ia (SNe Ia) subsamples (carefully chosen to coincide with the cluster positions). The influence of the different $\mathrm{SNe}$ Ia light-curve fitters in the previous analysis are also discussed.

Methods. We assumed that $\eta$ is a function of the redshift parametrized by two different relations: $\eta(z)=1+\eta_{0} z$, and $\eta(z)=$ $1+\eta_{0} z /(1+z)$, where $\eta_{0}$ is a constant parameter quantifying the possible departure from the strict validity of the DD relation. In order to determine the probability density function (PDF) of $\eta_{0}$, we considered the angular diameter distances from galaxy clusters recently studied by two different groups by assuming elliptical and spherical isothermal $\beta$ models and spherical non-isothermal $\beta$ model. The strict validity of the DD relation will occur only if the maximum value of $\eta_{0}$ PDF is centered on $\eta_{0}=0$.

Results. For both approaches we find that the elliptical $\beta$ model agrees with the distance-duality relation, whereas the non-isothermal spherical description is, in the best scenario, only marginally compatible. We find that the two-light curve fitters (SALT2 and MLCS2K2) present a statistically significant conflict, and a joint analysis involving the different approaches suggests that clusters are endowed with an elliptical geometry as previously assumed.

Conclusions. The statistical analysis presented here provides new evidence that the true geometry of clusters is elliptical. In principle, it is remarkable that a local property such as the geometry of galaxy clusters might be constrained by a global argument like the one provided by the cosmological distance-duality relation.
\end{abstract}

Key words. X-rays: galaxies: clusters - cosmic background radiation - distance scale

\section{Introduction}

The reciprocity law or reciprocity theorem, proved long ago by Etherington (1933), is a fundamental keystone for the interpretation of astronomical observations in cosmology. It states that if source and observer are in relative motion, solid angles subtended between the source and the observer are related by geometrical invariants where the redshift $z$ measured for the source by the observer enters in the relation. The core idea of this law comes from the invariance of various geometrical properties when there is a transposition between the roles of source and observer in astronomical observations. Proofs were presented in the context of relativistic geometrical optics, where it comes as a consequence of the geodesic deviation equation, as well as in the context of relativistic kinetic theory, where it is based on the Liouville integral for collision-free photons. The fundamental hypothesis behind the reciprocity law is the one made in General Relativity that light travels along null geodesics in a Riemannian spacetime (see Ellis 1971, 2007, and references therein).
Etherington's reciprocity law can be presented in various ways, either in terms of solid angles or relating various cosmological distances. Its most useful version in the context of astronomical observations, sometimes referred to as the distanceduality (DD) relation, relates the luminosity distance, $D_{\mathrm{L}}$, with the angular diameter distance, $D_{\mathrm{A}}$, by means of the following equation

$\frac{D_{\mathrm{L}}}{D_{\mathrm{A}}}(1+z)^{-2}=1$.

Since this result is easily proved in Friedmann-LemaîtreRobertson-Walker (FLRW) cosmologies, perhaps this is the reason why the generality of the relation above is not fully appreciated by most authors. Indeed, this law is completely general, valid for all cosmological models based on Riemannian geometry, being dependent neither on Einstein field equations nor on the nature of matter. Therefore, the DD relation is valid for both homogeneous and inhomogeneous cosmological models, requiring only that source and observer are connected by null 
geodesics in a Riemannian spacetime and that the number of photons is conserved.

The DD relation plays an essential role in modern cosmology, ranging from gravitational lensing studies (Schneider et al. 1992) to analyses of galaxy distribution and galaxy clusters observations (Lima et al. 2003; Cunha et al. 2007; Rangel Lemos \& Ribeiro 2008; Ribeiro 1992, 1993, 2005; Ribeiro \& Stoeger 2003; Albani et al. 2007; Mantz et al. 2010; Komatsu et al. 2011), as well as the plethora of cosmic consequences from primary and secondary temperature anisotropies of the cosmic microwave blackbody radiation (CMBR) observations (Komatsu et al. 2011). Other consequences of Etherington's reciprocity relation are the temperature shift equation $T_{\mathrm{o}}=T_{\mathrm{e}} /(1+z)$, where $T_{\mathrm{o}}$ is the observed temperature and $T_{\mathrm{e}}$ is the emitted temperature, a key result for analyzing CMBR observations, as well as the optical theorem that surface brightness of an extended source does not depend on the angular diameter distance of the observer from the source, an important result for understanding lensing brightness (Ellis 2007). In this connection, we also observe that any source of attenuation, such as "gray" intergalactic dust or exotic photon interaction, contributes to violate the DD relation because its proof is based on the conservation of the average number of photons.

Although taken for granted in virtually all analyses in cosmology, Eq. (1) is in principle testable by means of astronomical observations (Uzan et al. 2004; Basset \& Kunz 2004; Holanda et al. 2010; Li et al. 2011; Nair et al. 2011). If one is able to find cosmological sources whose intrinsic luminosities (standard candles) as well as their intrinsic sizes (standard rulers) are known, one can determine both $D_{\mathrm{L}}$ and $D_{\mathrm{A}}$ and, after measuring the redshifts, test the cosmic version of Etherington's result as given by the equation above. Note that ideally both quantities must be measured in a way that does not utilize any relationship coming from a cosmological model, that is, they must be determined by means of intrinsic astrophysically measured quantities. Therefore, the ideal way of observationally testing the reciprocity Eq. (1) would require independent measurements of intrinsic luminosities and sizes of sources, that is, independent determinations of $D_{\mathrm{A}}$ and $D_{\mathrm{L}}$ for a given set of sources.

There are, however, less-than-ideal methods to test Eq. (1). These usually assume a cosmological model suggested by a set of observations, apply this model in the context of some astrophysical effect, thereby trying to see if the DD relation remains valid. In this context, Uzan et al. (2004) argued that the SunyaevZel'dovich effect plus X-ray techniques for measuring $D_{\mathrm{A}}(z)$ from galaxy clusters (Sunyaev \& Zel'dovich 1972; Cavaliere \& Fusco-Fermiano 1978) is strongly dependent on the validity of this relation (see details in the next section). Briefly, in the context of this phenomenon one may consider the different electronic density dependencies combined with some assumptions about the galaxy cluster morphology in order to evaluate its angular diameter distance with basis on Eq. (1), such that

$D_{\mathrm{A}}(z) \propto \frac{D_{\mathrm{A}}^{2}\left(\Delta T_{0}\right)^{2} \Lambda_{e H 0}}{D_{\mathrm{L}}{ }^{2} S_{\mathrm{X} 0} T_{e 0}{ }^{2}} \frac{1}{\theta_{\mathrm{c}}} \propto \frac{\left(\Delta T_{0}\right)^{2} \Lambda_{e H 0}}{(1+z)^{4} S_{\mathrm{X} 0} T_{e 0}{ }^{2}} \frac{1}{\theta_{\mathrm{c}}}$,

where $S_{\mathrm{X} 0}$ is the central X-ray surface brightness, $T_{e 0}$ is the central temperature of the intra-cluster medium, $\Lambda_{e H O}$ is the central $\mathrm{X}$-ray cooling function of the intra-cluster medium, $\Delta T_{0}$ is the central decrement temperature, and $\theta_{\mathrm{c}}$ refers to a characteristic scale of the cluster along the line of sight (l.o.s.), whose exact meaning depends on the assumptions adopted to describe the galaxy cluster morphology.
On the other hand, in order to test the validity of DD relation it is convenient to assume a deformed expression:

$\frac{D_{\mathrm{L}}}{D_{\mathrm{A}}}(1+z)^{-2}=\eta(z)$

and from Eq. (2) we have (see Sect. 4 for details)

$D_{\mathrm{A}}^{\text {data }}(z)=D_{\mathrm{A}}(z) \eta(z)^{2}$,

actually, multiplied by $\eta^{-2}$ in the notation of Uzan et al. (2004). This quantity is reduced to the standard angular diameter distance only when the DD relation is strictly valid $(\eta \equiv 1)$. In order to quantify the $\eta$ parameter, Uzan et al. (2004) fixed $D_{\mathrm{A}}(z)$ by using the cosmic concordance model (Spergel et al. 2003) while for $D_{\mathrm{A}}^{\text {data }}(z)$ they considered the 18 galaxy clusters from the Reese et al. (2002) sample for which a spherically symmetric cluster geometry has been assumed. By assuming $\eta$ constant, their statistical analysis provided $\eta=0.91_{-0.04}^{+0.04}(1 \sigma)$, and is therefore only marginally consistent with the standard result.

Basset \& Kunz (2004) used supernovae Ia (SNe Ia) data as measurements of the luminosity distance and the estimated $D_{\mathrm{A}}$ from FRIIb radio galaxies (Daly \& Djorgovski 2003) and ultra compact radio sources (Gurvitz 1994, 1999; Lima \& Alcaniz 2000, 2002; Santos \& Lima 2008) in order to test possible new physics based on the following generalization of Eq. (1)

$\frac{D_{\mathrm{L}}(z)}{D_{\mathrm{A}}(z)(1+z)^{2}}=(1+z)^{\beta-1} \exp \left[\gamma \int_{0}^{z} \frac{\mathrm{d} z^{\prime}}{E\left(z^{\prime}\right)\left(1+z^{\prime}\right)^{\alpha}}\right]$,

where $E(z) \equiv H(z) / H_{0}$ is the dimensionless Hubble expansion, a quantity normalized to unity today. Note that for arbitrary values of $\alpha$, the strict validity of the DD relation corresponds to $(\beta, \gamma)=(1,0)$. By marginalizing on $\Omega_{\mathrm{M}}, \Omega_{\Lambda}$ and Hubble parameters, Basset \& Kunz (2004) found a $2 \sigma$ violation caused by excess brightening of SNIa at $z>0.5$, perhaps owing to a lensing magnification bias.

On the other hand, De Bernardis et al. (2006) also confronted the angular distances from galaxy clusters with luminosity distance data from SNe Ia to obtain a model-independent test. In order to compare the data sets they considered the weighted average of the data in seven bins and found that $\eta=1$ is consistent on a $68 \%$ confidence level $(1 \sigma)$. However, one needs to be careful when using the Sunyaev-Zel'dovich effect together with $\mathrm{X}$-ray techniques for measuring angular diameter distances to test the DD relation because this technique also depends on its validity. Indeed, when the relation does not hold, $D_{\mathrm{A}}(z)$ determined from observations is in general $D_{\mathrm{A}}^{\text {data }}(z)=D_{\mathrm{A}}(z) \eta^{2}$, which is reduced to $D_{\mathrm{A}}$ only if $\eta=1$. This means that De Bernardis and co-workers did not really test the DD relation, at least not in a consistent way. In addition, these authors binned their data, and, as such, their results may have been influenced by the particular choice of redshift binning.

Avgoustidis et al. (2010) also adopted an extended DD relation, $D_{\mathrm{L}}=D_{\mathrm{A}}(1+z)^{2+\epsilon}$, in the context of a flat $\Lambda \mathrm{CDM}$ model for constraining the cosmic opacity. The recent SN type Ia data compilation (Kowalski et al. 2008) was combined with the latest measurements of the Hubble expansion at redshifts in the range $0<z<2$ (Stern et al. 2010). They found $\epsilon=-0.04_{-0.07}^{+0.08}(2 \sigma)$. It should be stressed, however, that the main goal of the quoted studies was to merely test the consistency between the assumed cosmological model and the results provided by a chosen set of astrophysical phenomena.

Below we explore a different route to test the DD relation by using two complementary, but independent, approaches. First, 
we take the DD's validity for granted in order to access the galaxy cluster morphology. The basic idea is very simple and may be described as follows. The usually assumed spherical geometry of clusters has been severely questioned after some analyses based on data from the XMM-Newton and Chandra satellites, which suggested that clusters are supposed to exhibit an elliptical surface brightness. In this context, by assuming the $\Lambda \mathrm{CDM}$ framework (WMAP7), we discuss the constraints coming from the validity of DD relation on the local physics, that is, when different assumptions about the cluster geometry are considered. In order to do that, we considered three samples of angular diameter distances from galaxy clusters obtained through Sunyaev-Zel'dovich effect and X-ray measurements. These samples differ by the assumptions adopted to describe the clusters: (i) isothermal elliptical; (ii) isothermal spherical $\beta$ models (De Filippis et al. 2005), and non-isothermal spherical double $\beta$ model (Bonamente et al. 2006). Secondly, we propose a consistent cosmological-model-independent test for the DD relation by using subsamples of SNe Ia carefully chosen from Constitution data (Hicken et al. 2009) and the angular diameter distances from galaxy clusters. These topics were partially discussed by us (Holanda et al. 2010, 2011) without considering the second possibility (isothermal spherical $\beta$ model) that has also been analyzed by De Filippis et al. (2005). Both approaches were separately investigated, however, by avoiding all details of the Sunyaev-Zel'dovich effect and X-ray cluster physics.

In this article, we intend to close the above described gaps by providing a closer discussion of the physics involved, and, for completeness, we also included the spherical $\beta$ model case. In addition, the influence of the different SNe Ia light-curve fitters on the model-independent test involving the Sunyaev-Zel'dovich effect, X-ray and SNe Ia is discussed. Finally, a joint analysis involving the different approaches is also investigated. The present study (based on complementary tests) suggests that clusters are endowed with an elliptical geometry as assumed by De Filippis et al. (2005), once the strict validity of DD relation is taken for granted.

The paper is organized as follows. Sunyaev-Zel'dovich effect and X-ray surface brightness observations as a test for the DD relation are explored in Sect. 2. The galaxy cluster samples used in this paper are presented in Sect. 3. The consistence between the validity of the DD relation and the different assumptions used to describe the galaxy clusters usually adopted in the literature are discussed in Sect. 4. In Sect. 5 we discuss a new and modelindependent cosmological test for the DD relation involving luminosity distances from $\mathrm{SNe}$ Ia and $D_{\mathrm{A}}(z)$ from galaxy clusters. In Sect. 6 we study the influence of the different SNe Ia lightcurve fitters on the results of the previous section, while the joint analysis is presented in 7. Finally, the main conclusions and future prospects are summarized in Sect. 8.

\section{SZE/X-ray technique and the distance-duality relation}

An important phenomenon occurring in galaxy clusters is the Sunyaev-Zel'dovich effect (SZE), a small distortion of the CMBR spectrum provoked by the inverse Compton scattering of the CMBR photons passing through a population of hot electrons. The SZE is proportional to the electron pressure integrated along the 1.o.s., i.e., to the first power of the plasma density. The measured temperature decrement $\Delta T_{\mathrm{SZ}}$ of the $\mathrm{CMBR}$ is given by (De Filippis et al. 2005)

$\frac{\Delta T_{\mathrm{SZ}}}{T_{\mathrm{CMBR}}}=f\left(v, T_{\mathrm{e}}\right) \frac{\sigma_{\mathrm{T}} k_{\mathrm{B}}}{m_{\mathrm{e}} c^{2}} \int_{\text {l.o.s. }} n_{\mathrm{e}} T_{\mathrm{e}} \mathrm{d} l$, where $T_{\mathrm{e}}$ is the temperature of the intra-cluster medium, $k_{\mathrm{B}}$ the Boltzmann constant, $T_{\mathrm{CMBR}}=2.728^{\circ} \mathrm{K}$ is the temperature of the CMBR, $\sigma_{\mathrm{T}}$ the Thompson cross section, $m_{\mathrm{e}}$ the electron mass and $f\left(v, T_{\mathrm{e}}\right)$ accounts for frequency shift and relativistic corrections (Itoh et al. 1998; Nozawa et al. 1998).

Other important physical phenomena occurring in the intragalaxy cluster medium are the X-ray emission caused by thermal bremsstrahlung and line radiation resulting from electronion collisions. The X-ray surface brightness $S_{\mathrm{X}}$ is proportional to the integral along the line of sight of the square of the electron density. This quantity may be written as follows

$S_{\mathrm{X}}=\frac{D_{\mathrm{A}}^{2}}{4 \pi D_{\mathrm{L}}^{2}} \int_{\text {l.o.s. }} n_{\mathrm{e}}^{2} \Lambda_{e H} \mathrm{~d} l$,

where $\Lambda_{e H}$ is the X-ray cooling function of the intra-cluster medium (measured in the cluster rest frame) and $n_{\mathrm{e}}$ is the electron number density. It thus follows that the SZE and X-ray emission both depend on the properties $\left(n_{\mathrm{e}}, T_{\mathrm{e}}\right)$ of the intra cluster medium.

As is well known, it is possible to obtain the angular diameter distance from galaxy clusters by their SZE and X-ray surface brightness observations. The calculation begins by constructing a model for the cluster gas distribution. Assuming, for instance, the spherical isothermal $\beta$-model such that $n_{\mathrm{e}}$ is given by (Cavaliere \& Fusco-Fermiano 1978)

$n_{\mathrm{e}}(r)=\left(1+\frac{r^{2}}{r_{\mathrm{c}}^{2}}\right)^{-3 \beta / 2}$,

Eqs. (6) and (7) can be integrated. Here $r_{\mathrm{c}}$ is the core radius of the galaxy cluster. This $\beta$ model is based on the hydrostatic equilibrium equation and constant temperature (Sarazin 1988). In this way, we may write for the SZE

$\Delta T_{\mathrm{SZ}}=\Delta T_{0}\left(1+\frac{\theta^{2}}{\theta_{\mathrm{c}}^{2}}\right)^{1 / 2-3 \beta / 2}$,

where $\theta_{\mathrm{c}}=r_{\mathrm{c}} / D_{\mathrm{A}}$ is the angular core radius and $\Delta T_{0}$ is the central temperature decrement that includes all physical constants and terms resulting from the line-of-sight integration. More precisely:

$\Delta T_{0} \equiv T_{\mathrm{CMBR}} f\left(v, T_{\mathrm{e}}\right) \frac{\sigma_{\mathrm{T}} k_{\mathrm{B}} T_{\mathrm{e}}}{m_{\mathrm{e}} c^{2}} n_{e 0} \sqrt{\pi} \theta_{\mathrm{c}} D_{\mathrm{A}} g(\beta / 2)$,

with

$g(\alpha) \equiv \frac{\Gamma[3 \alpha-1 / 2]}{\Gamma[3 \alpha]}$,

where $\Gamma(\alpha)$ is the gamma function and the others constants are the usual physical quantities. For X-ray surface brightness, we have

$S_{\mathrm{X}}=S_{\mathrm{X} 0}\left(1+\frac{\theta}{\theta_{\mathrm{c}}^{2}}\right)^{1 / 2-3 \beta}$,

where the central surface brightness $S_{\mathrm{X} 0}$ reads

$S_{\mathrm{X} 0} \equiv \frac{D_{\mathrm{A}}^{2} \Lambda_{e H} \mu_{\mathrm{e}} / \mu_{\mathrm{H}}}{D_{\mathrm{L}}^{2} 4 \sqrt{\pi}} n_{e 0}^{2} \theta_{\mathrm{c}} D_{\mathrm{A}} g(\beta)$.

Here $\mu$ is the molecular weight given by $\mu_{i} \equiv \rho / n_{i} m_{\mathrm{p}}$.

One can solve Eqs. (10) and (13) for the angular diameter distance by eliminating $n_{e 0}$ and taking for granted the validity of 
DD relation. However, a more general result appears when the DD relation is not regarded as being strictly valid. In this case one obtains

$$
\begin{aligned}
D_{\mathrm{A}}= & {\left[\frac{\Delta T_{0}^{2}}{S_{\mathrm{X} 0}}\left(\frac{m_{\mathrm{e}} c^{2}}{k_{\mathrm{B}} T_{e 0}}\right)^{2} \frac{g(\beta)}{g(\beta / 2)^{2} \theta_{\mathrm{c}}}\right] } \\
& \times\left[\frac{\Lambda_{e H 0} \mu_{\mathrm{e}} / \mu_{\mathrm{H}}}{4 \pi^{3 / 2} f\left(v, T_{\mathrm{e}}\right)^{2}\left(T_{\mathrm{CMBR}}\right)^{2} \sigma_{\mathrm{T}}^{2}\left(1+z_{\mathrm{c}}\right)^{4}} \frac{1}{\eta(z)^{2}}\right] \\
= & D_{\mathrm{A}}^{\text {data }} \eta^{-2},
\end{aligned}
$$

where $z_{\mathrm{c}}$ is the galaxy cluster redshift. Therefore, as previously stressed by Uzan et al. (2004), galaxy cluster observations do not provide the angular diameter distance directly. In principle, instead of the real angular diameter distance, the measured quantity is $D_{\mathrm{A}}^{\text {data }}(z)=D_{\mathrm{A}}(z) \eta^{2}(z)$.

To proceed with our analysis, the quantity $\eta(z)$ defining the deformed DD relation (see Eq. (3)) is parametrically described by two different expressions (Holanda et al. 2010, 2011)

i) $\eta(z)=1+\eta_{0} z$, ii) $\eta(z)=1+\eta_{0} z /(1+z)$.

The first expression is a continuous and smooth one-parameter linear expansion, whereas the second one includes a possible epoch-dependent correction that avoids the divergence at extremely high $z$. Naturally, one may argue that these relations were not derived from first principles. However, we stress that these expressions are very simple and have several advantages such as a manageable one-dimensional phase space and a good sensitivity to observational data. Clearly, the second parametrization can also be rewritten as $\eta(z)=1+\eta_{0}(1-a)$, where $a(z)=(1+z)^{-1}$ is the cosmic-scale factor. It represents an improvement with respect to the linear parametrization, since the DD relation becomes bounded regardless of the redshift values. Potentially, it will become more useful once higher redshift cluster data are made available.

The above parametrizations are clearly inspired by similar expressions for the $\omega(z)$-equation of state parameter in timevarying dark energy models (see, for instance, Padmanabhan \& Choudury 2003; Linder 2003; Cunha et al. 2007; Silva et al. 2007). In the limit of very low redshifts $(z \ll 1)$, we have $\eta=1$ and $D_{\mathrm{L}}=D_{\mathrm{A}}$ as should be expected, and, more important for our subsequent analysis, the value $\eta_{0}=0$ must be favored by the Etherington principle. In other words, for a given data set, the likelihood of $\eta_{0}$ must be peaked at $\eta_{0}=0$, in order to satisfy the Etherington theorem. It should be remarked that Gonçalves et al. (2012) also adopted these expressions to explored the DD relation by using observations of gas mass fractions of galaxy clusters, whereas Lima et al. (2011) showed how to derive them from first principles based on possible theoretical modifications of the luminosity distance (without refraction effects).

\section{Galaxy cluster samples}

Below, the physical constraints encoded in the possibility of a deformed DD relation is explored by considering three samples of angular diameter distances from galaxy clusters obtained by combining their SZE and X-ray surface brightness observations. The first is defined by 38 angular diameter distances from galaxy clusters in the redshift range $0.14 \leq z \leq 0.89$, as given in the Bonamente et al. (2006) sample, where the hydrostatic equilibrium model and spherical symmetry was considered to describe the galaxy clusters. In order to construct a realistic model for the cluster gas distribution and include the possible presence of the cooling flow, these authors modeled the gas density with a function whose form is given below (Bonamente et al. 2006; La Roque et al. 2006),

$n_{\mathrm{e}}(r)=n_{e 0} \cdot\left[f\left(1+\frac{r^{2}}{r_{\mathrm{c} 1}^{2}}\right)^{-\frac{3 \beta}{2}}+(1-f)\left(1+\frac{r^{2}}{r_{\mathrm{c} 2}^{2}}\right)^{-\frac{3 \beta}{2}}\right]$.

This double $\beta$-model for the density generalizes the single $\beta$-model profile, introduced by Cavaliere \& Fusco-Fermiano (1976) and the double $\beta$ model proposed by Mohr et al. (1999). It has the freedom of following both the central spike in density and the more gentle outer distribution. The quantity $n_{e 0}$ is the central density, $f$ governs the fractional contributions of the narrow and broad components $(0 \leq f \leq 1), r_{\mathrm{c} 1}$ and $r_{\mathrm{c} 2}$ are the two core radii that describe the shape of the inner and outer portions of the density distribution and $\beta$ determines the slope at large radii (the same $\beta$ is used for both the central and outer distributions in order to reduce the total number of degrees of freedom).

On the other hand, the hydrostatic equilibrium and spherical symmetry hypotheses result in the condition

$\frac{\mathrm{d} P}{\mathrm{~d} r}=-\rho_{\mathrm{g}} \frac{\mathrm{d} \phi}{\mathrm{d} r}$,

where $P$ is the gas pressure, $\rho_{\mathrm{g}}$ is the gas density and $\phi=$ $-G M(r) / r$ is the gravitational potential due to dark matter and the plasma. Using the ideal gas equation of state for the diffuse intracluster plasma, $P=\rho_{\mathrm{g}} k_{\mathrm{B}} T / \mu m_{\mathrm{p}}$, where $\mu$ is the mean molecular weight and $m_{\mathrm{p}}$ is the proton mass, one obtains a relationship between the cluster temperature and the cluster mass distribution,

$$
\begin{aligned}
\frac{\mathrm{d} T}{\mathrm{~d} r} & =-\left(\frac{\mu m_{\mathrm{p}}}{k_{\mathrm{B}}} \frac{\mathrm{d} \phi}{\mathrm{d} r}+\frac{T}{\rho_{\mathrm{g}}} \frac{\mathrm{d} \rho_{\mathrm{g}}}{\mathrm{d} r}\right), \\
& =-\left(\frac{\mu m_{\mathrm{p}}}{k_{\mathrm{B}}} \frac{G M}{r^{2}}+\frac{T}{\rho_{\mathrm{g}}} \frac{\mathrm{d} \rho_{\mathrm{g}}}{\mathrm{d} r}\right) .
\end{aligned}
$$

Bonamente et al. (2006) combined hydrostatic equilibrium equations with a dark matter density distribution from Navarro, Frenk \& White (1997),

$\rho_{\mathrm{DM}}(r)=\mathcal{N}\left[\frac{1}{\left(r / r_{\mathrm{s}}\right)\left(1+r / r_{\mathrm{s}}\right)^{2}}\right]$,

where $\mathcal{N}$ is a density normalization constant and $r_{\mathrm{s}}$ is a scale radius. The parameters of these equations $\left(n_{e 0}, f, r_{\mathrm{c} 1}, r_{\mathrm{c} 2}, \beta, \mathcal{N}\right.$ and $r_{\mathrm{s}}$ ) were obtained by calculating the joint likelihood $\mathcal{L}$ of the X-ray and SZE data in a Markov chain Monte Carlo method (Bonamente et al. 2004). Summarizing, the cluster plasma and dark matter distributions were analyzed assuming hydrostatic equilibrium model and spherical symmetry, thereby accounting for radial variations in density, temperature and abundance.

The second sample is formed by 25 galaxy clusters in the redshift range $0.023 \leq z \leq 0.8$ compiled by De Filippis et al. (2005). These authors re-analyzed archival X-ray data of the XMM-Newton and Chandra satellites of two samples for which combined X-ray and SZE analysis have already been reported using an isothermal spherical $\beta$-model. One of the samples, compiled previously by Reese et al. (2002), is a selection of 18 galaxy clusters distributed over the redshift interval $0.14<z<0.8$. The other one, the sample of Mason et al. (2001), has seven clusters from the X-ray limited flux sample of Ebeling et al. (1996). In this way, De Filippis et al. (2005) used an isothermal elliptical $\beta$-model and an isothermal spherical $\beta$ model to obtain $D_{\mathrm{A}}(z)$ measurements for these galaxy 
R. F. L. Holanda et al.: Probing cosmic distance duality relation
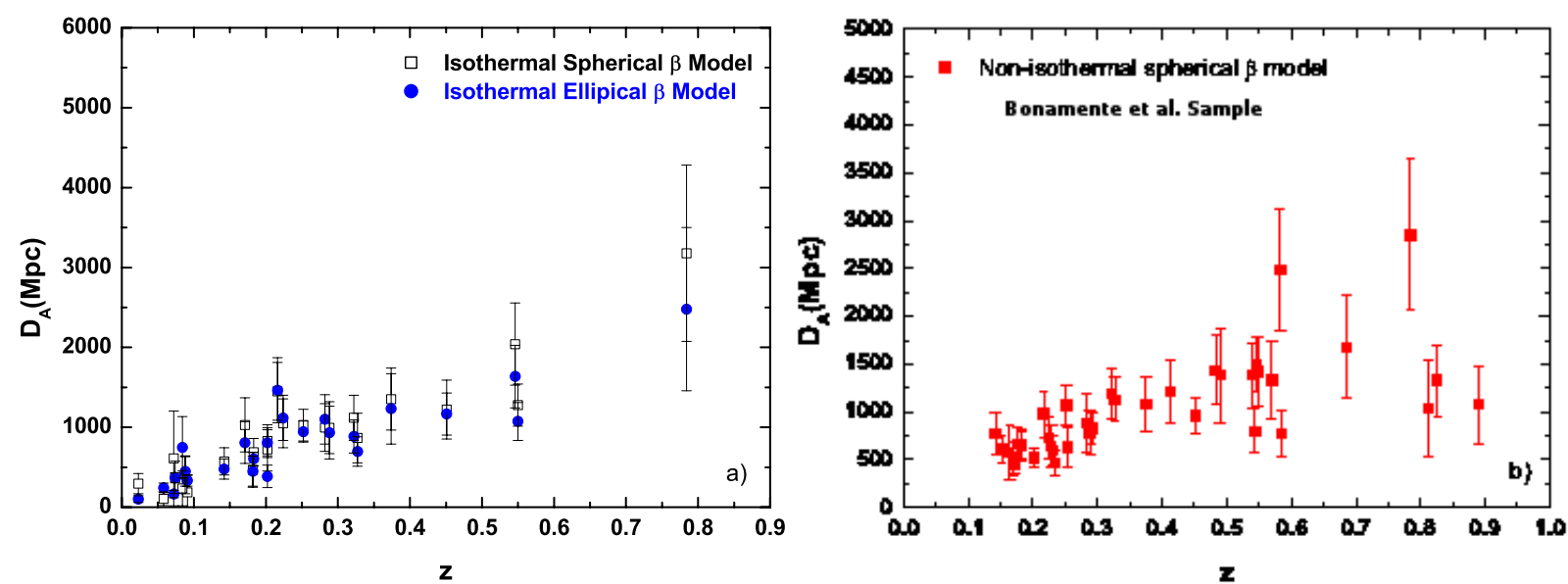

Fig. 1. (Color online) a) Filled (blue) circles and open (black) squares with the associated error bars (only statistical errors) stand for the De Filippis et al. (2005) samples: isothermal elliptical $\beta$ model and isothermal spherical $\beta$ model, respectively. b) The sample of Bonamente et al. (2006) where a non-isothermal spherical $\beta$ model was used to describe the galaxy clusters.

clusters samples. As discussed by De Filippis et al. (2005), the choice of circular rather than elliptical $\beta$ model does not affect the resulting central surface brightness or Sunyaev-Zeldovich decrement, the slope $\beta$ differs slightly between these models, but significantly different values for core radius are obtained. The result was that the core radius of the elliptical $\beta$-model is bigger than that of the spherical $\beta$ model (see Fig. 1 in their paper). In a first approximation it was found that $\theta_{\text {ell }}=$ $\frac{2 e_{\text {proj }}}{1+e_{\text {proj }}} \theta_{\text {circ }}$, where $e_{\text {proj }}$ is the axial ratio of the major to the minor axes of the projected isophotes. Since $D_{\mathrm{A}} \propto \theta_{\mathrm{c}}^{-1}$, angular diameter distances obtained by using an isothermal spherical $\beta$-model are overestimated compared with those from the elliptical $\beta$-model.

For the isothermal elliptical $\beta$-model De Filippis et al. (2005) used a general triaxial morphology to describe the intra-cluster medium. They obtained

$\Delta T_{\mathrm{SZ}}=\Delta T_{0}\left[1+\frac{\theta_{1}^{2}+\left(e_{\mathrm{proj}}\right)^{2} \theta_{2}^{2}}{\left(\theta_{\mathrm{c}, \text { proj }}\right)^{2}}\right]^{1 / 2-3 \beta / 2}$,

where

$$
\begin{aligned}
\Delta T_{0} \equiv & T_{\mathrm{CMBR}} f\left(v, T_{\mathrm{e}}\right) \frac{\sigma_{\mathrm{T}} k_{\mathrm{B}} T_{\mathrm{e}}}{m_{\mathrm{e}} c^{2}} n_{e 0} \sqrt{\pi} \\
& \times \frac{D_{\mathrm{A}} \theta_{\mathrm{c}, \text { proj }}}{h^{3 / 4}} \sqrt{\frac{e_{1} e_{2}}{e_{\text {proj }}}} g(\beta / 2),
\end{aligned}
$$

$g(\alpha)$ is given by Eq. (11), $D_{\mathrm{A}}$ is the angular diameter distance to the cluster, $\theta_{i} \equiv x_{i, \text { obs }} / D_{\mathrm{A}}$ is the projected angular position (on the plane of the sky) of the intrinsic orthogonal coordinate $x_{i, \mathrm{obs}}, h$ is a function of the cluster shape and orientation, $e_{\text {proj }}$ is the axial ratio of the major to the minor axes of the observed projected isophotes and $\theta_{\mathrm{c} \text {, proj }}$ is the projection on the plane of the sky (p.o.s.) of the intrinsic angular core radius.

Similarly, the X-ray surface brightness $S_{\mathrm{X} 0}$ can be written as follows,

$S_{\mathrm{X}}=S_{\mathrm{X} 0}\left[1+\frac{\theta_{1}^{2}+\left(e_{\mathrm{proj}}\right)^{2} \theta_{2}^{2}}{\left(\theta_{\mathrm{c}, \text { proj }}\right)^{2}}\right]^{1 / 2-3 \beta}$,

where the central surface brightness $S_{\mathrm{X} 0}$ reads

$S_{\mathrm{X} 0} \equiv \frac{\Lambda_{e H} D_{\mathrm{A}}^{2}\left(\mu_{\mathrm{e}} / \mu_{\mathrm{H}}\right)}{4 \sqrt{\pi} D_{\mathrm{L}}^{2}} n_{e 0}^{2} \frac{D_{\mathrm{A}} \theta_{\mathrm{c}, \text { proj }}}{h^{3 / 4}} \sqrt{\frac{e_{1} e_{2}}{e_{\text {proj }}}} g(\beta)$,

and $\mu$ is the molecular weight, given by $\mu_{i} \equiv \rho / n_{i} m_{\mathrm{p}}$.
By eliminating $n_{\mathrm{e} 0}$ in the equations above and assuming the DD relation as valid, i.e., $D_{\mathrm{A}}^{\text {data }}(z)=D_{\mathrm{A}}(z)$, De Filippis et al. (2005) obtained the observational quantity as written below

$$
\begin{aligned}
D_{\mathrm{A}}(z)= & \frac{\Delta T_{0}^{2}}{S_{\mathrm{X} 0}}\left(\frac{m_{\mathrm{e}} c^{2}}{k_{\mathrm{B}} T_{e 0}}\right)^{2} \frac{g(\beta)}{g(\beta / 2)^{2} \theta_{\mathrm{c}, \text { proj }}} \\
& \times \frac{\Lambda_{e H 0}\left(\mu_{\mathrm{e}} / \mu_{\mathrm{H}}\right)}{4 \pi^{3 / 2} f\left(v, T_{\mathrm{e}}\right)^{2}\left(T_{\mathrm{CMBR}}\right)^{2} \sigma_{\mathrm{T}}^{2}\left(1+z_{\mathrm{c}}\right)^{4}} .
\end{aligned}
$$

The slope $\beta$ of the profile and the projected core radius $\theta_{\mathrm{c} \text {, proj }}$ were obtained by fitting the cluster surface brightness with an elliptical 2-D $\beta$ model. For the isothermal spherical $\beta$ model description, De Filippis et al. (2005) considered the usual Eq. (8) and obtained angular distances with Eq. (14) and $\eta=1$.

In Fig. 1 we plot the galaxy cluster samples. In Fig. 1a the filled circles (blue) and open squares (black) with the associated error bars (only statistical errors) stand for the De Filippis et al. (2005) isothermal elliptical $\beta$ model and isothermal spherical $\beta$ model, respectively. In Fig. 1b we show the sample of Bonamente et al. (2006), where a non-isothermal spherical double $\beta$ model was used to describe the galaxy clusters (red filled squares).

\section{Obtaining the shape of galaxy clusters by using the $\mathrm{DD}$ relation as constraint}

Many studies about the intra-cluster gas and dark matter (DM) distribution in galaxy clusters have been limited to the standard spherical geometry (Reiprich \& Boringer 2002; Bonamente et al. 2006; Shang et al. 2009). However, in the past few years observations of galaxy clusters based on Chandra and XMM data have shown that generally clusters exhibit elliptical surface brightness maps. Simulations have also predicted that DM halos show axis ratios typically on the order of $\approx 0.8$ (Wang \& White 2009), thereby disproving the spherical geometry assumption. In this line, the first determination of the intrinsic three-dimensional (3D) shapes of galaxy clusters was presented by Morandi et al. (2010) by combining X-ray, weak-lensing and strong-lensing observations. Their methodology was applied to the galaxy cluster MACS J1423.8+2404 and they found a triaxial galaxy cluster geometry with DM halo axial ratios $1.53 \pm 0.15$ and $1.44 \pm 0.07$ on the plane of the sky and along the line of sight, respectively.

Bearing in mind these results, we propose a new method to access the galaxy cluster morphology by taking the validity of 
the DD relation as a constraint. The idea is very simple. Beacuse the samples shown in 3 were compiled under different geometric assumptions, we confront these underlying hypotheses with the validity of the DD relation. In principle, this kind of result provides an interesting example of how a cosmological (global) condition correlates to the local physics. In the application, one should also keep in mind that a deformed DD relation as given by Eq. (3) naturally induces a more general result for the angular diameter distance from galaxy clusters via SZE and X-ray technique (see Eq. (14))

$D_{\mathrm{A}}^{\text {data }}(z)=D_{\mathrm{A}}(z) \eta^{2}$.

In this line, our aim is to estimate the $\eta_{0}$ parameter for each galaxy cluster sample for both parameterizations of $\eta(z)$ as given by Eq. (15). In our analyses (Holanda et al. 2011), $D_{\mathrm{A}}(z)$ for each galaxy clusters is obtained from the WMAP (seven years) results by fixing the conventional flat $\Lambda \mathrm{CDM}$ model. The parameters of the simplest six-parameter $\Lambda \mathrm{CDM}$ model were recently determined by Komatsu et al. (2011) by using the combination of seven-year data from WMAP with the latest distance measurements from baryon acoustic oscillations (BAO) in the distribution of galaxies (Percival et al. 2010) and the Hubble parameter $H_{0}$ measurement presented by Riess et al. (2009). The basic results are $\Omega_{\Lambda}=0.725 \pm 0.016$ and $h=0.702 \pm 0.014$, and no convincing evidence for deviations from the minimal cosmic concordance model has been established.

The theoretical angular diameter distance can be written as (Lima et al. 2003; Cunha et al. 2007)

$D_{\mathrm{A}}\left(z ; h, \Omega_{\mathrm{m}}\right)=\frac{3000 h^{-1}}{(1+z)} \int_{o}^{z} \frac{\mathrm{d} z^{\prime}}{\mathcal{H}\left(z^{\prime} ; \Omega_{\mathrm{m}}\right)} \mathrm{Mpc}$,

where $h=H_{0} / 100 \mathrm{~km} \mathrm{~s}^{-1} \mathrm{Mpc}^{-1}$ and the dimensionless function $\mathcal{H}\left(z^{\prime} ; \Omega_{\mathrm{m}}\right)$ is given by

$\mathcal{H}=\left[\Omega_{\mathrm{m}}\left(1+z^{\prime}\right)^{3}+\left(1-\Omega_{\mathrm{m}}\right)\right]^{1 / 2}$.

Now, in order to constrain $\eta_{0}$, let us evaluate the likelihood distribution function, $\mathrm{e}^{-\chi^{2} / 2}$, where

$\chi^{2}=\sum_{z} \frac{\left\{[\eta(z)]^{2}-\left[\eta_{\mathrm{obs}}(z)\right]^{2}\right\}^{2}}{\sigma_{\eta_{\mathrm{obs}}}^{2}}$

with $\left[\eta_{\mathrm{obs}}^{2}(z)\right]=D_{\mathrm{A}}^{\mathrm{data}}(z) / D_{\mathrm{A}}(z)$ and

$\sigma_{\eta_{\mathrm{obs}}}^{2}=\left[\frac{1}{D_{\mathrm{A}}(z)}\right]^{2} \sigma_{\mathrm{data}}^{2}+\left[\frac{D_{\mathrm{A}}^{\mathrm{data}}}{D_{\mathrm{A}}^{2}(z)}\right]^{2} \sigma_{\mathrm{WMAP}}^{2}$,

where $\sigma_{\text {WMAP }}^{2}$ is the error in $D_{\mathrm{A}}(z)$ associated to cosmological parameters. The common statistical error contributions for galaxy clusters are SZE point sources $\pm 8 \%$, X-ray background $\pm 2 \%$, Galactic $N_{\mathrm{H}} \leq \pm 1 \%, \pm 15 \%$ for cluster asphericity, $\pm 8 \%$ kinetic SZ and for CMBR anisotropy $\leq \pm 2 \%$. On the other hand, the estimates for systematic effects are SZ calibration $\pm 8 \%$, $\mathrm{X}$-ray flux calibration $\pm 5 \%$, radio halos $+3 \%$, and X-ray temperature calibration $\pm 7.5 \%$. Indeed, one may show that typical statistical errors amount to nearly $20 \%$, in agreement with other works (Mason et al. 2001; Reese et al. 2002, 2004), while for systematics we also find typical errors around $+12.4 \%$ and $-12 \%$ (see also Table 3 in Bonamente et al. 2006). In the present analysis we have combined the statistical and systematic errors in quadrature for the galaxy clusters $\left(\sigma_{\text {data }}^{2}=\sigma_{\text {stat }}^{2}+\sigma_{\text {syst }}^{2}\right)$. We
Table 1. The $\eta_{0}$ values and their errors (statistical + systematic errors) of our first analysis (Sect. 4).

\begin{tabular}{lc}
\hline \hline Isothermal elliptical $\beta$ model $\times \Lambda \mathrm{CDM}(\mathrm{WMAP})$ & $\chi^{2} /$ d.o.f \\
\hline$\eta_{0}($ Linear case $)=-0.056 \pm-0.10(1 \sigma)$ & $23.52 / 24$ \\
$\eta_{0}($ Non-linear case $)=-0.088 \pm 0.14(1 \sigma)$ & $22.56 / 24$ \\
Isothermal spherical $\beta$ model $\times \Lambda \mathrm{CDM}(\mathrm{WMAP} 7)$ & $\chi^{2} /$ d.o.f \\
$\eta_{0}($ Linear case $)=0.19 \pm 0.12(1 \sigma)$ & $20.16 / 24$ \\
$\eta_{0}($ Non-linear case $)=0.28 \pm 0.18(1 \sigma)$ & $19.44 / 24$ \\
Non-isothermal spherical $\beta$ model $\times \Lambda \mathrm{CDM}($ WMAP7) & $\chi^{2} /$ d.o.f \\
$\eta_{0}$ (Linear case $)=-0.11 \pm 0.06(1 \sigma)$ & $29.6 / 37$ \\
$\eta_{0}$ (Non-linear case) $=-0.16 \pm 0.08(1 \sigma)$ & $29.23 / 37$ \\
\hline
\end{tabular}

note that in our $\chi^{2}$ statistical analysis the asymmetric uncertainties present in the Bonamente et al. (2006) and De Filippis et al. (2005) samples were symmetrized by using the D'Agostini (2004) method.

In Figs. $2 \mathrm{a}$ and $\mathrm{b}$ we plot the likelihood distribution function for the De Filippis et al. (2005) and Bonamente et al. (2006) samples. The $\eta_{0}$ values and their errors (statistical + systematic errors) of our analysis are given below (see Table 1).

For an isothermal elliptical $\beta$ model, we see that the angular diameter distances from the De Filippis et al. (2005) sample provide an excellent fit and agree with the DD relation at $1 \sigma$ confidence level. On the other hand, although it agrees with the DD relation at $2 \sigma$ c.l., the analysis based on the isothermal spherical $\beta$ model leads to $\eta_{0}$ values higher than those from the elliptical $\beta$ case. Since in this case $\eta_{0}>0$, the departures of the DD relation validity indicates that the estimated angular distances with the spherical $\beta$ model are overestimated with respect to those from the conventional flat $\Lambda \mathrm{CDM}$ (WMAP7). For the Bonamente et al. (2006) sample (non-isothermal spherical double $\beta$ model) we also see that the strict validity of the reciprocity relation is only marginally compatible. The relative situation is not modified even when only clusters with $z>0.1$ are considered in the De Filippis et al. (2005) sample. In such a situation, we obtain $\eta_{0}=-0.044_{-0.1}^{+0.1}\left(\chi^{2} /\right.$ d.o.f. $\left.=15.9 / 17\right)$ for the linear parametrization, and $\eta_{0}=-0.07_{-0.14}^{+0.14}\left(\chi^{2} /\right.$ d.o.f. $\left.=15.6 / 17\right)$ within $1 \sigma$ in the non-linear case for elliptical description and $\eta_{0}=0.186_{-0.1}^{+0.11}\left(\chi^{2} /\right.$ d.o.f. $\left.=10.9 / 17\right)$ for the linear parametrization, and $\eta_{0}=0.274_{-0.145}^{+0.145}\left(\chi^{2} /\right.$ d.o.f. $\left.=10.7 / 17\right)$ within $1 \sigma$ in the non-linear case for spherical description.

Therefore, we found no evidence for a distance-duality violation for the elliptical De Filippis et al. (2005) sample. However, the same kind of analysis contradicts the spherical symmetry hypothesis assumed in the Bonamente et al. (2006) sample and in the De Filippis et al. (2005) sample when a spherical geometry is assumed. These results are very interesting since they show how important the choice of geometry is in describing the clusters to obtain their distances through SZE + X-ray measurements. We also see that the non-isothermal assumption of Bonamente et al. (2006) in their spherical description was not sufficient to satisfy the validity of the DD relation in the $\Lambda$ CDM framework (WMAP7, Komatsu et al. 2011).

We recall that Bonamente et al. (2006) used three different models to describe the same 38 galaxy clusters: (i) the nonisothermal spherical double $\beta$ model already discussed; (ii) an isothermal spherical $\beta$ model; and (iii) the isothermal spherical $\beta$ model excluding the central $100 \mathrm{kpc}$ from the X-ray data. For the sake of completeness, we also obtained the $\eta_{0}$ values for the last two descriptions. For an isothermal spherical $\beta$ model with linear and non-linear parametrizations we found $\eta_{0}=-0.09_{-0.06}^{+0.05}\left(\chi^{2} /\right.$ d.o.f. $\left.=48.48 / 37\right)$ and $\eta_{0}=-0.13_{-0.09}^{+0.08}$ 

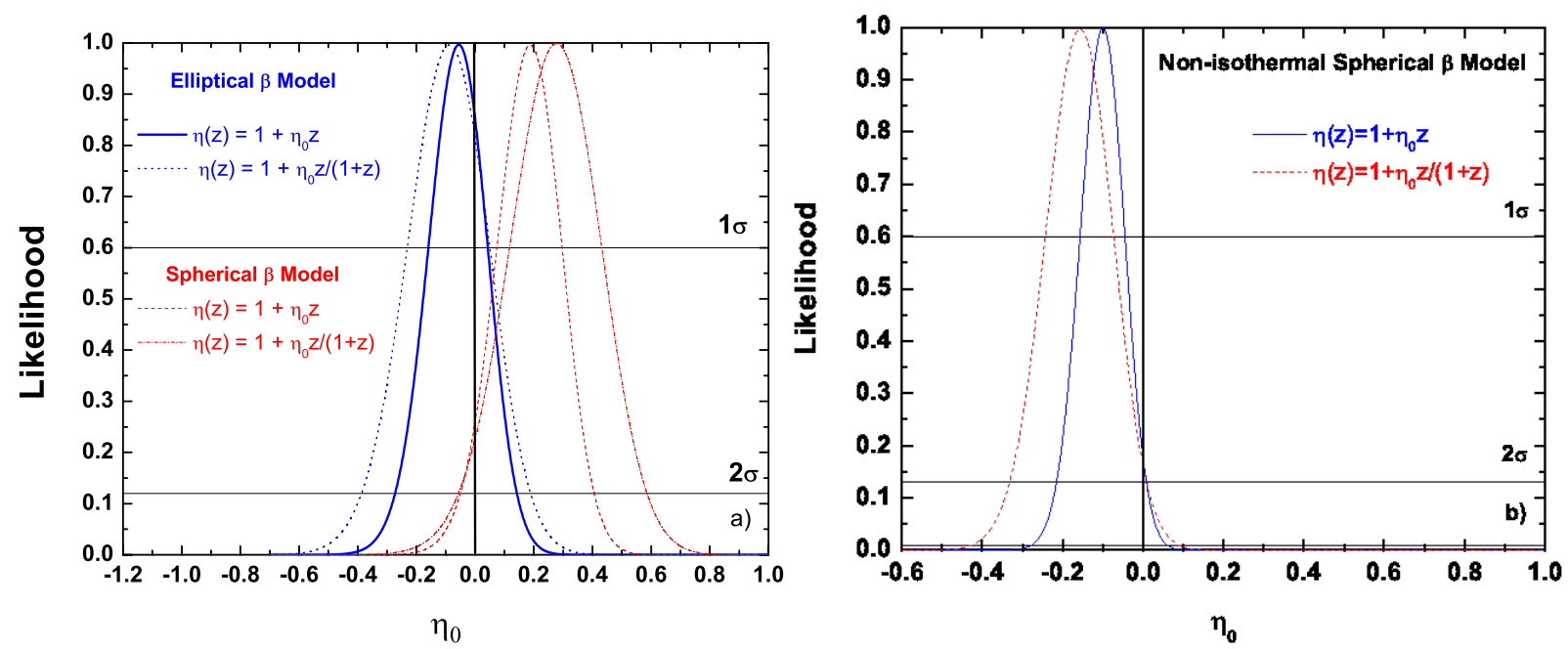

Fig. 2. (Color online) a) Likelihood distribution functions for De Filippis et al. (2005) sample. The solid and the dotted blue lines are likelihood functions corresponding to linear and non-linear $\eta(z)$ parametrizations for the isothermal elliptical $\beta$ model. The dashed and the dashed-dotted red lines are likelihood functions corresponding to linear and non-linear parametrizations for the isothermal spherical $\beta$ model. b) The likelihood distribution functions for Bonamente et al. (2006) sample. The solid blue line and the dotted red line correspond to linear and non-linear parametrizations.

$\left(\chi^{2} /\right.$ d.o.f. $\left.=47.56 / 37\right)$, respectively. In the isothermal spherical $\beta$ model excluding the central $100 \mathrm{kpc}$ from the X-ray data we obtained $\eta_{0}=-0.14_{-0.07}^{+0.07}\left(\chi^{2} /\right.$ d.o.f. $\left.=47.81 / 37\right)$ for the linear parametrization, and $\eta_{0}=-0.2_{-0.11}^{+0.09}\left(\chi^{2} /\right.$ d.o.f. $\left.=48.40 / 37\right)$ in the non-linear case. All these determinations are at a $1 \sigma$ confidence level.

Furthermore, Bonamente et al. (2006) also determined the Hubble constant $H_{0}$ for each model. By assuming a flat $\Lambda$ CDM model $\left(\Omega_{\mathrm{M}}=0.3\right.$ and $\left.\Omega_{\Lambda}=0.7\right)$, the corresponding values at $1 \sigma$ c.l. are $H_{0}=76.9_{-3.4}^{+3.9}, H_{0}=73.7_{-3.8}^{+4.6}$ and $H_{0}=77.6_{-4.3}^{+4.8}$. More recently, Cunha et al. (2007) obtained $H_{0}=74 \pm 3.5$ through a joint analysis with BAO by using the De Filippis et al. sample (2005), thereby alleviating the tension between between $\mathrm{SZ}+\mathrm{X}$-ray technique and the $\mathrm{CMB}+\mathrm{BAO}$ determination of $H_{0}$. However, since the Hubble constants obtained from all these models agree at $1 \sigma$, one may conclude that the $H_{0}$ value is not useful to decide which galaxy cluster model is more realistic.

\section{Testing the DD relation with galaxy clusters and SNe la}

In this section we apply a model-independent cosmological test for the DD relation based on the three samples defined in Sect. 3. For $D_{\mathrm{L}}$ we considered two subsamples of SNe type Ia taken from Tables 1 and 2 of the Hicken et al. (2009) (Constitution data), whereas values for $D_{\mathrm{A}}$ are provided by the three samples of galaxy clusters discussed above. The SNe Ia redshifts of each subsample were carefully chosen to coincide with those of the associated galaxy cluster sample $(\Delta z<0.005)$, thereby allowing a direct test of the DD relation. For a given pair of data sets (SNe Ia, galaxy clusters), one should expect a likelihood of $\eta_{0}$ peaked at $\eta_{0}=0$, in order to satisfy the DD relation. Moreover, in our approach the data do not need to be binned as assumed in some analyses involving the DD relation (see, for instance, De Bernardis et al. 2006).

In Fig. $3 \mathrm{a}$ we plot $D_{\mathrm{A}}$ multiplied by $\left(1+z_{\text {cluster }}\right)^{2}$ from the galaxy clusters sample compiled from the De Filippis et al. (2005) sample and $D_{\mathrm{L}}$ from our first $\mathrm{SNe}$ Ia subsample. In Fig. 3b we plot the subtraction of redshift between clusters and $\mathrm{SNe}$ Ia. We see that the biggest difference is $\Delta z \approx 0.01$ for three
Table 2. The ( $\left.\eta_{0}\right)$ values and their errors (statistical + systematic errors) of our second analysis (section 5).

\begin{tabular}{lc}
\hline \hline Isothermal elliptical $\beta$ model $\times \mathrm{SNe}$ Ia & $\chi^{2} /$ d.o.f \\
\hline$\eta_{0}($ Linear case $)=-0.28 \pm 0.21(1 \sigma)$ & $21.2 / 21$ \\
$\eta_{0}($ Non-linear case $)=-0.43 \pm 0.29(1 \sigma)$ & $21 / 21$ \\
Isothermal spherical $\beta$ model $\times \mathrm{SNe}$ Ia & $\chi^{2} /$ d.o.f \\
$\eta_{0}($ Linear case $)=0.14 \pm 0.26(1 \sigma)$ & $17.01 / 21$ \\
$\eta_{0}($ Non-linear case $)=0.20 \pm 0.36(1 \sigma)$ & $16.80 / 21$ \\
Non-isothermal spherical $\beta$ model $\times \mathrm{SNe}$ Ia & $\chi^{2} /$ d.o.f \\
$\eta_{0}($ Linear case $)=-0.39 \pm 0.11(1 \sigma)$ & $30.26 / 34$ \\
$\eta_{0}($ Non-linear case $)=-0.61 \pm 0.16(1 \sigma)$ & $28.9 / 34$ \\
\hline
\end{tabular}

clusters (open squares), while for the remaining 22 clusters we have $\Delta z<0.005$. In order to avoid the corresponding bias, we removed the three clusters from all subsequent analyses so that $\Delta z<0.005$ for all pairs.

Similarly, in Fig. 4a we plot $D_{\mathrm{A}}$ multiplied by $\left(1+z_{\text {cluster }}\right)^{2}$, but now for the Bonamente et al. sample (2006) and $D_{\mathrm{L}}$ from our second SNe Ia subsample. In Fig. 4b we display the redshift subtraction between clusters and SNe Ia. Again, we see that for 35 clusters $\Delta z<0.005$. The biggest difference is again $\Delta z \approx 0.01$ for three clusters, and, for consistency, these were also removed from our analysis.

Let us now estimate the parameter $\eta_{0}$ for each sample and the two parametrizations defined by Eq. (3). We recall that in general the SZE + X-ray surface brightness observations technique does not produce $D_{\mathrm{A}}(z)$, but $D_{\mathrm{A}}^{\text {data }}(z)=D_{\mathrm{A}}(z) \eta^{2}$. Consequently, if one wishes to test Eq. (1) with SZE + X-ray observations from galaxy clusters, the angular diameter distance must be replaced by $D_{\mathrm{A}}^{\text {data }}(z) \eta^{-2}$ in Eq. (3). In this way, we obtained $\eta(z)=D_{\mathrm{A}}^{\text {data }}(z)\left(1+z_{\text {cluster }}\right)^{2} / D_{\mathrm{L}}(z)$.

Following standard procedure, the likelihood estimator is determined by a $\chi^{2}$ statistics

$\chi^{2}=\sum_{z} \frac{\left[\eta(z)-\eta_{\mathrm{obs}}(z)\right]^{2}}{\sigma_{\eta_{\mathrm{obs}}}^{2}}$, 

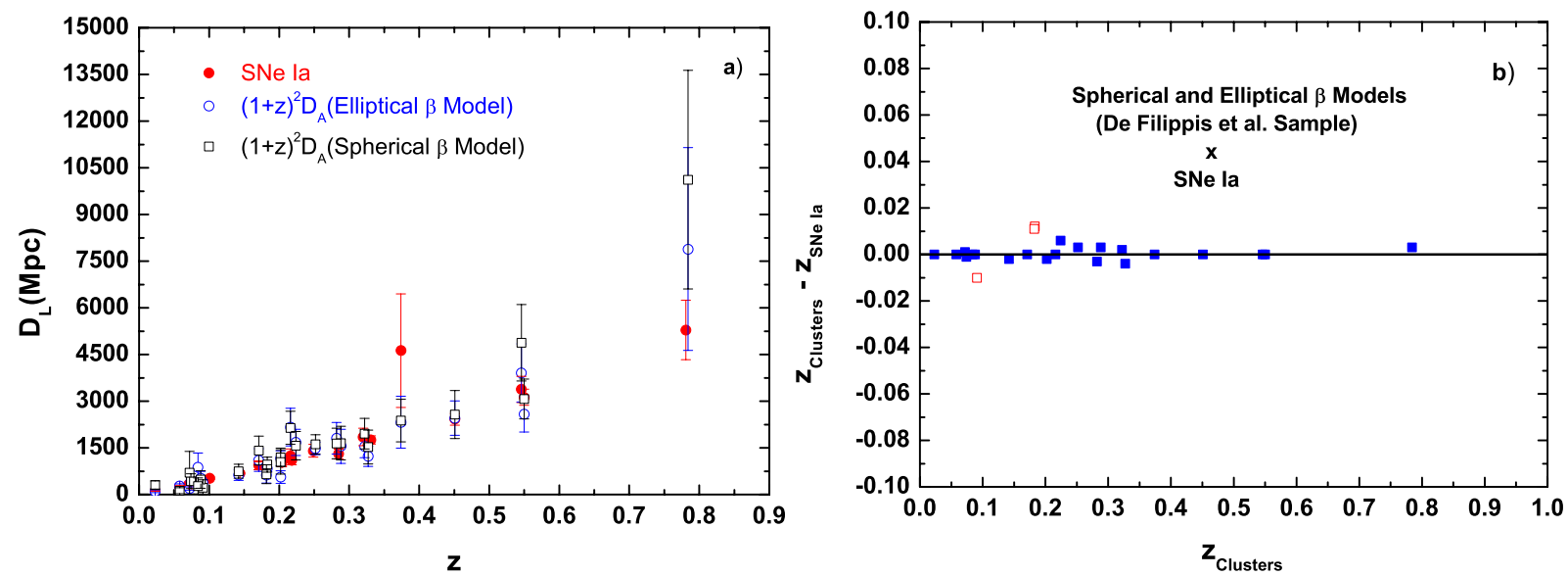

Fig. 3. (Color online) a) Galaxy clusters and SNe Ia data. The open blue, open black, and filled red circles with the associated error bars stand for the galaxy clusters described with elliptical and spherical $\beta$ models of the De Filippis et al. (2005) (statistical + systematical errors) and SNe Ia (only statistical errors) samples, respectively. b) The redshift subtraction for the same pair of cluster-SNe Ia samples. The open squares represent the pairs of points for which $\Delta z \approx 0.01$.
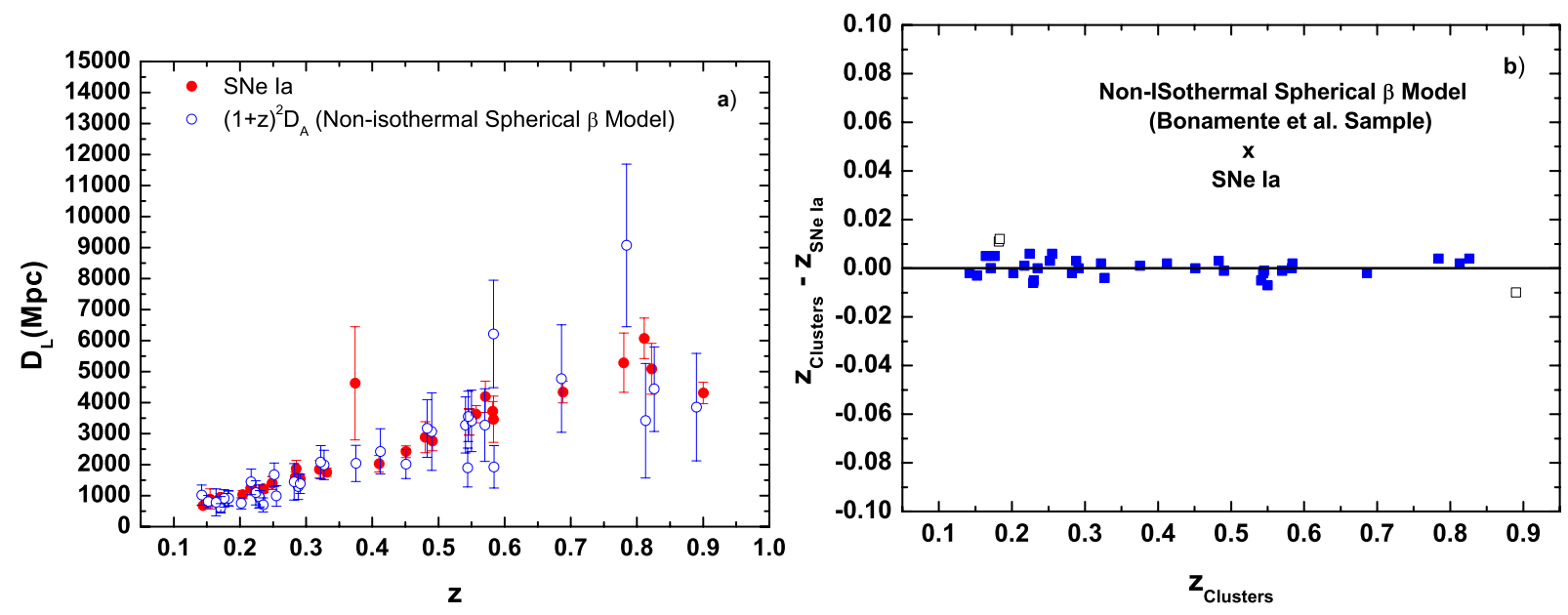

Fig. 4. (Color online) a) Galaxy clusters and SNe Ia data. The open (blue) and filled (red) circles with the associated error bars stand for the Bonamente et al. (2006) (statistical + systematical errors) and SNe Ia (only statistical errors) samples, respectively. b) The redshift subtraction for the same pair of cluster-SNe Ia samples. As in Fig. 1b, the open squares represent the pairs of points with the biggest difference in redshifts $(\Delta z \approx 0.01)$.

where $\eta_{\mathrm{obs}}(z)=\left(1+z_{\text {cluster }}\right)^{2} D_{\mathrm{A}}^{\mathrm{data}}(z) / D_{\mathrm{L}}(z)$ and

$\sigma_{\eta_{\mathrm{obs}}}^{2}=\left[\frac{\left(1+z_{\text {cluster }}\right)^{2} D_{\mathrm{A}}^{\text {data }}}{D_{\mathrm{L}}^{2}}\right]^{2} \sigma_{D_{\mathrm{L}}}^{2}+\left[\frac{\left(1+z_{\text {cluster }}\right)^{2}}{D_{\mathrm{L}}}\right]^{2} \sigma_{D_{\mathrm{A}}^{\text {data }}}^{2}$

As in the previous section, we combined the statistical and systematic errors in quadrature for the angular diameter distance from galaxy clusters, and, as we remarked above, the asymmetric error bars were treated by the D'Agostini (2004) method.

On the other hand, after nearly 500 discovered SNe Ia, the constraints on the cosmic parameters from luminosity distance are now limited by systematics rather than by statistical errors. In principle, there are two main sources of systematic uncertainties in SNe Ia observations, which are closely related to photometry and possible corrections for light-curve shape (Hicken at al. 2009). However, at the moment it is not so clear how one is to estimate the overall systematic effects for these standard candles (Komatsu et al. 2011), and, therefore, we exclude them from the following analysis. The basic reason is that systematic effects from galaxy clusters seem to be larger than those of SNe observations, but their inclusion does not affect the results validity of the distance-duality relation very much. In this way, following
Holanda et al. (2010), we included only statistical errors of the $\mathrm{SNe}$ Ia magnitude measurements.

In Figs. 5a and b we plot the likelihood distribution function for each sample. The $\eta_{0}$ values with their errors (statistical and systematic errors) are provided in Table 2. Clearly, the confrontation between the angular diameter distances from the elliptical De Filippis et al. (2005) sample with SNe Ia data disagree moderately with the reciprocity relation (the DD relation is marginally satisfied in $2 \sigma$ ) and the $\eta_{0}$ values are mostly negative. Since $\left(1+z_{\text {cluster }}\right)^{2} D_{\mathrm{A}}^{\text {data }}(z) / D_{\mathrm{L}}(z) \propto 1+\eta_{0} z$, negative $\eta_{0}$ values indicate luminosity distances overestimated with respect to the angular diameter ones. This tension between Sne Ia and the elliptical De Filippis et al. (2005) sample arises because $\mathrm{SNe}$ Ia samples prefer universes with a higher $\Omega_{\Lambda}$ value than those of the WMAP7 results. Indirectly, our analysis support the tension found by Wei (2010), who used SNe Ia constitution data and observations of the CMBR anisotropy plus BAO separately to constrain the $\omega_{a}$ parameter of the dark energy equation of state given by $\omega=\omega_{0}+\omega_{a} z /(1+z)$. The $\mathrm{SNe}$ Ia constitution data indicated a strong negative $\omega_{a}\left(\omega_{a} \approx-11\right)$ and the phantom energy divide line $(\omega<-1)$ could be crossed at recent redshifts. 
R. F. L. Holanda et al.: Probing cosmic distance duality relation
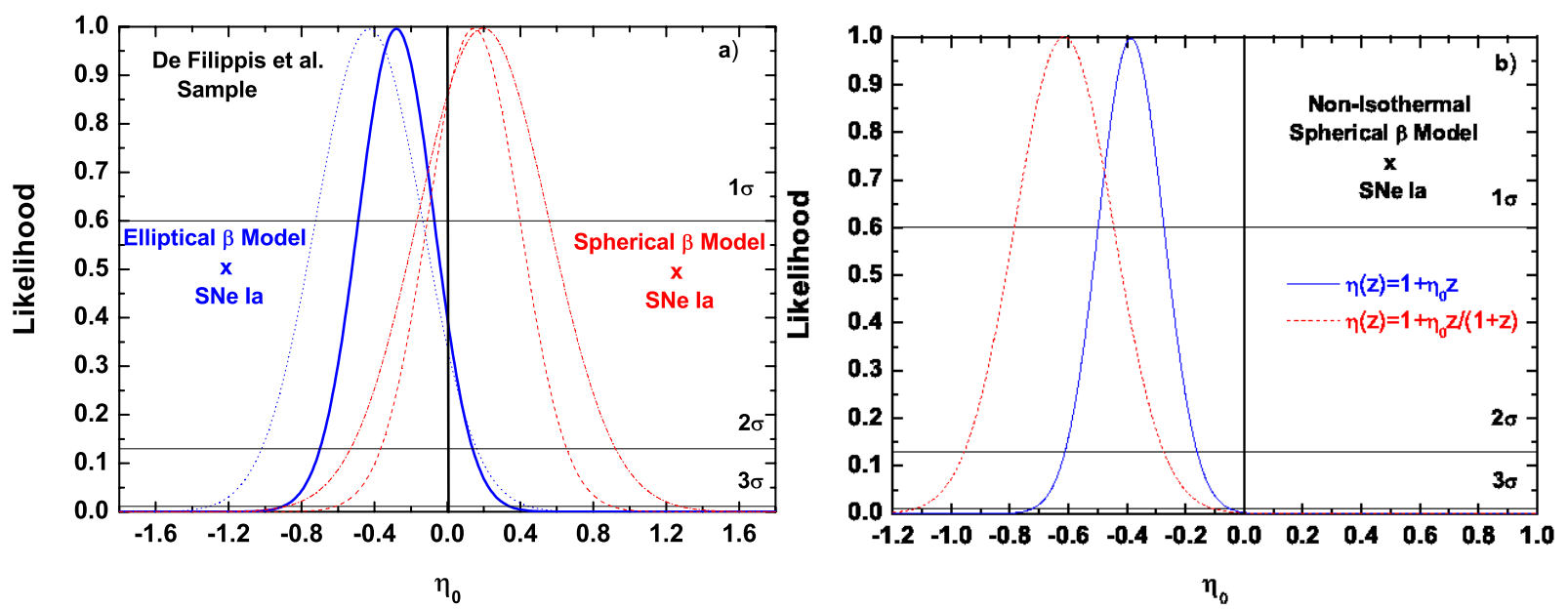

Fig. 5. (Color online) a) Likelihood distribution functions for De Filippis et al. (2005) sample. The solid and the dotted blue lines are likelihood functions for linear and non-linear parametrizations corresponding to the isothermal elliptical $\beta$ model. The dashed and the dashed-dotted red lines are likelihood functions for linear and non-linear parametrizations corresponding to the isothermal spherical $\beta$ model. b) The likelihood distribution functions for Bonamente et al. (2006) sample.

Furthermore, the constitution data disagreed not only with the $\mathrm{CMB}$ and BAO observations, but also with other SNe Ia datasets such as Davis (Davis et al. 2007) and SNLS (Astier et al. 2006). On the other hand, when an isothermal spherical $\beta$ model is used, the DD relation is satisfied in $1 \sigma$. As discussed earlier, this concordance occurs because an isothermal spherical $\beta$ model yields angular distances overestimated compared to the elliptical model and WMAP7 results. For the Bonamente et al. (2006) sample, where a non-isotermal spherical double $\beta$ model was assumed to describe the clusters, we see that the DD relation is not obeyed even at $3 \sigma$. We note that our results do not change if one replaces $z_{\text {cluster }}$ by $z_{\mathrm{SNe}}$, where $z_{\mathrm{SNe}}$ is the redshift of SNe Ia. This shows that a $\Delta z<0.005$ is sufficient to implement our direct test.

In this context, we recall that $\mathrm{Li}$ et al. (2011) rediscussed this independent cosmological test using the latest Union2 SNe Ia data and the angular diameter distances from galaxy clusters. However, they removed six and twelve data points, respectively, from the De Filippis et al. (2005) and Bonamente et al. (2006) samples and obtained a more serious violation of the DD relation. These authors also reexamined the DD relation by postulating two more general parameterization forms, namely $\eta(z)=$ $\eta_{0}+\eta_{1} z$ and $\eta(z)=\eta_{0}+\eta_{1} z /(1+z)$, and they found that consistencies between the observations and the DD relation are markedly improved for both samples of galaxy clusters. Nair et al. (2011) also discussed the strict validity of the DD relation using the latest Union2 SNe Ia data and the angular diameter distances from galaxy clusters, FRIIb radio galaxies and mock data. They proposed six different (one and two indices) parametrizations (including, as particular cases, those adopted by Holanda et al. 2010) in an attempt to determine a possible redshift variation of the DD relation. As physically expected, their results depend both on the specific parametrization and the data samples considered. In particular, they conclude that one index parameterization, namely $\eta_{V}=\eta_{8} /(1+z)$ and $\eta_{V}=\eta_{9} \exp \{[z /(1+z)] /(1+z)\}$, do not support the DD relation for the given data set.

More recently, Meng et al. (2012) also reinvestigated the model-independent test by comparing two different methods and several parametrizations (one and two indices) for $\eta(z)$. Their basic conclusion agrees with our studies in the sense that the triaxial ellipsoidal model is suggested by the model-independent test at $1 \sigma$ while the spherical $\beta$ model can only be accommodated at a $3 \sigma$ confidence level. As we shall see, this uncertainty can also be robustly resolved by considering a joint analysis involving the two treatments discussed here (see Sect. 7). In principle, since all results somewhat depend on the assumed $\eta(z)$ form, it is important to understand the effect of different parametrizations. However, we recall that at extremely low redshifts the DD relation reduces to unity since $D_{\mathrm{L}}(z) \equiv D_{\mathrm{A}}(z)$, and, therefore, the constant parameter appearing in the proposed $\eta(z)$ expressions should be fixed to unity.

At this point it is interesting to know the influence of $\mathrm{SNe}$ Ia light-curve fitters on the model-independent test of the distance duality relation and some of its consequences for the local physics. This topic has attracted a lot of attention in the recent literature (Kessler et al. 2009; Bengochea 2011; Hao Wei 2010), and its connection with the DD relation and galaxy cluster geometry it will be discussed next.

\section{SNe la light-curve fitters, DD relation and cluster geometries}

In the previous section, we tested the DD relation by using luminosity distances from $\mathrm{SNe}$ Ia based on the Constitution sample and the angular diameter distances from three galaxy clusters samples obtained by their SZE and X-rays surface brightness measurements. However, in the Constitution compilation different SNe Ia samples were analyzed by four light-curve fitters (SALT, SALT2, MLCS31 and MLCS17) to test consistency and systematic differences: 397 SNe Ia with SALT, 351 SNe Ia with SALT2, 366 SNe Ia with MLCS31 and 372 SNe Ia with MLCS17. The four fitters were seen to be relatively consistent with the light-curve-shape and color parameters, but that still leaves room for improvement, and they provide a considerable amount of systematic uncertainty to any analysis. In this way, it would be interesting to investigate the influence of different $\mathrm{SNe}$ Ia light-curve fitters on our previous results. Unfortunately, it was not possible to find the same SNe's Ia used here in Sect. 5 in the Constitution sample, but analyzed with the four methods for a direct comparison.

On the other hand, Kessler et al. (2009) presented the Hubble diagram for $103 \mathrm{SNe}$ Ia with redshifts $0.04<z<$ 0.42, discovered during the first season of the Sloan Digital Sky Survey-II (SDSS-II). These data filled the redshift desert between low- and high-redshift $\mathrm{SNe}$ Ia of the previous $\mathrm{SNe}$ Ia 

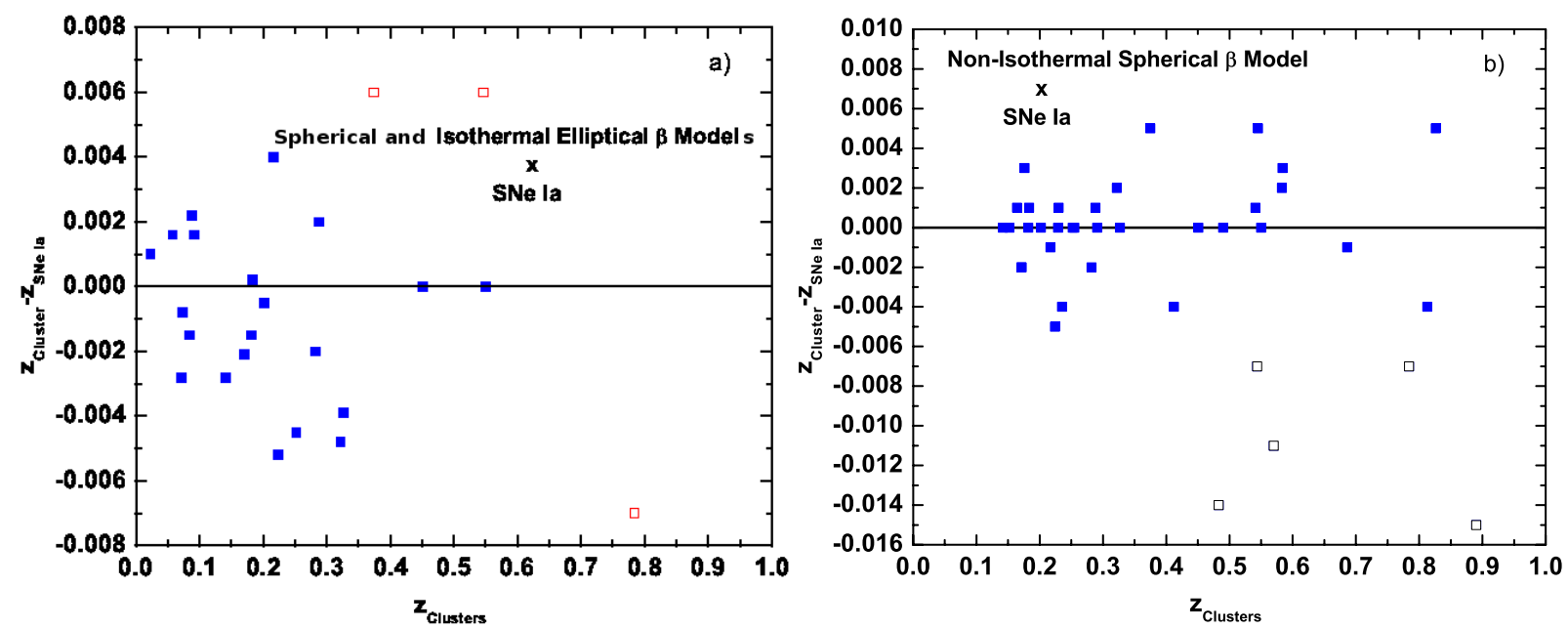

Fig. 6. (Color online) a) Redshift subtraction for the same pair of cluster-SNe Ia samples of De Filippis et al. (2005). The open squares represent the pairs of points for which $\Delta z>0.005$. b) The redshift subtraction for the same pair of cluster-SNe Ia samples for Bonamente et al. (2006). The open squares represent the pairs of points for which $\Delta z>0.005$.

surveys. In addition, these authors included a comprehensive and consistent reanalysis of other datasets (ESSENCE, SNLS, HST), resulting in a combined sample of $288 \mathrm{SNe}$ Ia. Reanalysis was performed by using two light-curve fitters: SALT2 (Guy et al. 2007) and MLCS2K2 (Jha et al. 2007). MLCS2K2 calibration uses a nearby training set of SNe Ia assuming a close to linear Hubble law, while SALT2 uses the whole data set to calibrate empirical light curve parameters, and a cosmological model must be assumed in this method. Typically a $\Lambda \mathrm{CDM}$ or a $\omega \mathrm{CDM}$ model is assumed. Consequently, the SNe Ia distance moduli obtained with SALT2 fitter retain a degree of model dependence (Bengochea 2011). Kessler et al. (2009) combined these 288 SNe Ia with measurements of baryon acoustic oscillations from the SDSS luminous red galaxy sample and with cosmic microwave background temperature anisotropy measurements from the WMAP for estimating the cosmological parameters $\omega$ and $\Omega_{\mathrm{M}}$ by assuming a spatially flat cosmological model. The results from these two light-curve fitters disagreed: $\omega=-0.76 \pm$ 0.07 (stat) \pm 0.11 (syst), $\Omega_{\mathrm{M}}=0.307 \pm 0.019$ (stat) \pm 0.023 (syst) using MLCS2K2 and $\omega=-0.96 \pm 0.06$ (stat) \pm 0.12 (syst), $\Omega_{\mathrm{M}}=0.265 \pm 0.016$ (stat) \pm 0.025 (syst) using SALT2. This discrepancy raised the question of which method is more reliable because it is not possible to definitively determine from the current data that either method is better or incorrect. The overall conclusion was that the cosmological parameter $\omega$ lies between -1.1 and -0.7 (Kessler et al. 2009). Exploring nonstandard cosmology scenarios, Sollerman et al. (2009) found that more exotic models provided a better fit to the SNe Ia data than the $\Lambda \mathrm{CDM}$ model when the MLCS2K2 light-curve fitter was used. However, when the SN Ia data were analyzed by using SALT2 light-curve fitter, the standard cosmological constant model agreed better to those data.

In this section, by using the SNe Ia data from SDSS-II (Kessler et al. 2009), we explore the influence of the two different SNe Ia light-curve fitters on our model-independent cosmological test for the DD relation. For $D_{\mathrm{L}}$ we considered two subsamples of SNe Ia taken from SDSS-II (2007) where, again, the $\mathrm{SNe}$ Ia redshifts of each subsample were carefully chosen to coincide with those of the associated galaxy cluster sample $(\Delta z<$ 0.005), allowing a direct test of the DD relation. Furthermore, in this case, each subsample of SNe Ia was analyzed by the MLCS2K2 and SALT2 light-curve fitters separately. For $D_{\mathrm{A}}$ we used the angular diameter distance samples of galaxy clusters discussed above: the isothermal elliptical and spherical $\beta$ model samples from De Filippis et al. (2005) and the non-isothermal spherical double $\beta$ model sample from Bonamente et al. (2006).

In Fig. 6a we plot the subtraction of redshift between clusters and SNe Ia for the De Filippis et al. (2005) sample. We see that the biggest difference is $\Delta z \approx 0.007$ for three clusters (open squares), whereas for the remaining 22 clusters we have $\Delta z<0.005$. In order to avoid the corresponding bias, the three clusters were removed from all analyses presented here so that $\Delta z<0.005$ for all pairs. In Fig. 6b we display the redshift subtraction between clusters and SNe Ia for the Bonamente et al. (2006) sample. In this case we see that for 33 clusters $\Delta z<0.005$. The biggest difference is $\Delta z \approx 0.015$ for five clusters, and, for consistency, they were also removed from our analysis.

In Figs. 7a and $\mathrm{b}$ we plot the likelihood distribution function for the elliptical and spherical De Filippis et al. (2005) samples, respectively. The results for Bonamente et al. (2006) sample are shown in Fig. 8. Following Kessler et al. (2009) and Sollerman et al. (2009), we added in our analyses an additional intrinsic dispersion of 0.16 mag to the uncertainties output by the MLCS2K2 light-curve fitter and 0.14 mag for the SALT2 light-curve fitter. In Table 3 we display our results, where the errors are in $2 \sigma$ (statistical plus systematic errors).

Interestingly, we also obtained conflicting results between the two light-curve fitters. In our analysis involving the elliptical De Filippis et al. (2005) sample (for both light-curve fitters), the DD relation validity is obtained at $1 \sigma$ (c.l.) with the SALT2 method. However, it is only marginally compatible with MLC2K2 method because the $\eta_{0}$ values are considerably negative. This result points to an overestimated luminosity distances when SNe Ia are analyzed with the MLC2K2 method. This result is not surprising since the De Filippis et al. (2005) sample agrees with the DD relation validity in the context of the $\Lambda \mathrm{CDM}$ model (WMAP7) (see Sect. 4) and the SALT2 light-curve fitter also favors the same cosmology according to the analyses of Kessler et al. (2009) and Sollerman et al. (2009). Curiously, the best verification of the DD relation validity is obtained by confronting the spherical sample of De Filippis et al. (2005) with SNe Ia when the MLC2K2 light-curve fitter is adopted. This probably occurs because both distances are overestimated 
R. F. L. Holanda et al.: Probing cosmic distance duality relation
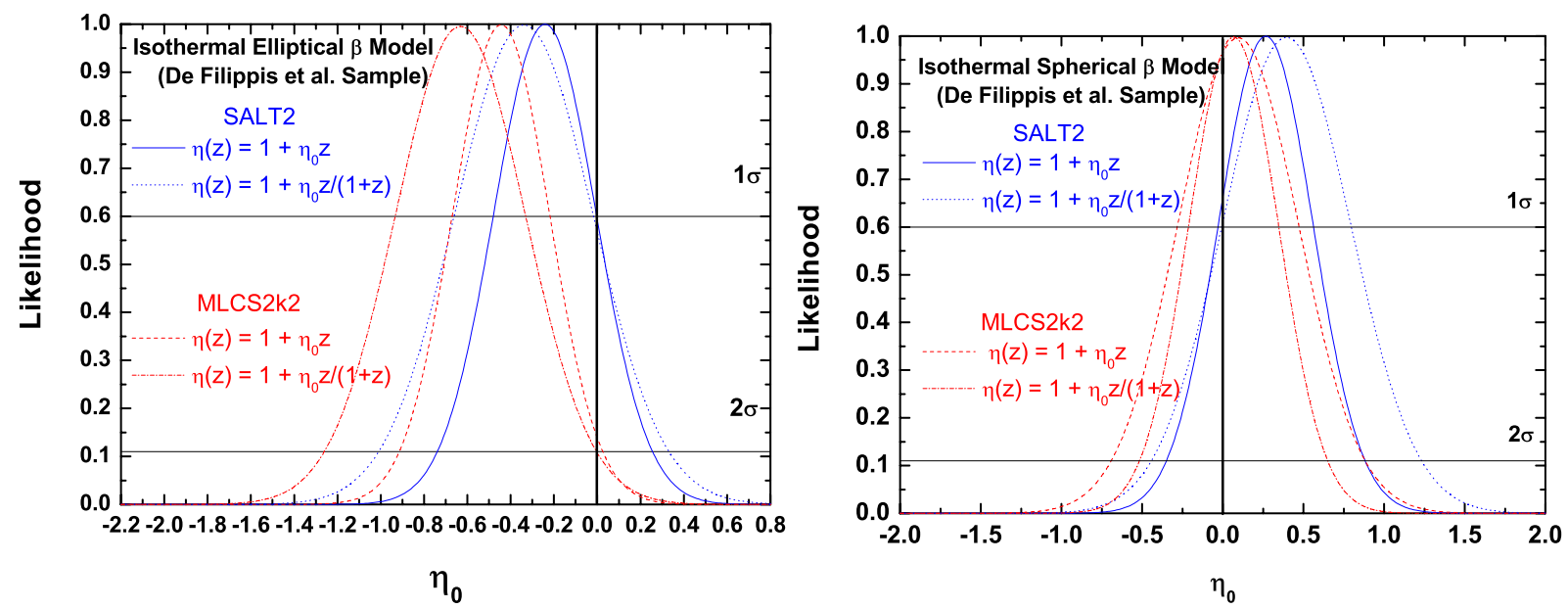

Fig. 7. (Color online) a) Likelihood distribution functions for the De Filippis et al. (2005) sample (elliptical $\beta$ model) for both $\eta(z)$ parametrizations and both SNe Ia light-curve fitters. b) The likelihood distribution functions for the De Filippis et al. (2005) sample (spherical $\beta$ model) for both $\eta(z)$ parametrizations and both SNe Ia light-curve fitters.

Table 3. The $\eta_{0}$ values for our third analysis (Sect. 6). All errors are at $1 \sigma$.

\begin{tabular}{lcccc}
\hline \hline Isothermal elliptical $\beta$ model & SALT2 & $\chi^{2} /$ d.o.f & MLCS2K2 & $\chi^{2} /$ d.o.f \\
\hline$\eta_{0}$ (Linear case) & $-0.24 \pm 0.24$ & $19.74 / 21$ & $-0.43 \pm 0.21$ & $21.2 / 21$ \\
$\eta_{0}$ (Non-linear case) & $-0.34 \pm 0.34$ & $19.53 / 21$ & $-0.63 \pm 0.32$ & $20.8 / 21$ \\
Isothermal spherical $\beta$ model & SALT2 & $\chi^{2} /$ d.o.f & MLCS2K2 & $\chi^{2} /$ d.o.f \\
$\eta_{0}$ (Linear case) & $0.27 \pm 0.27$ & $16 / 21$ & $0.01 \pm 0.34$ & $15.7 / 21$ \\
$\eta_{0}$ (Non-linear case) & $0.4 \pm 0.4$ & $15.7 / 21$ & $0.07 \pm 0.35$ & $15.5 / 21$ \\
Bonamente et al. sample & SALT2 & $\chi^{2} /$ d.o.f & MLCS2K2 & $\chi^{2} /$ d.o.f \\
$\eta_{0}$ (Linear case) & $-0.22 \pm 0.14$ & $21.76 / 32$ & $-0.28 \pm 0.10$ & $24 / 32$ \\
$\eta_{0}$ (Non-linear case) & $-0.28 \pm 0.24$ & $21.2 / 32$ & $-0.45 \pm 0.15$ & $23.7 / 32$ \\
\hline
\end{tabular}

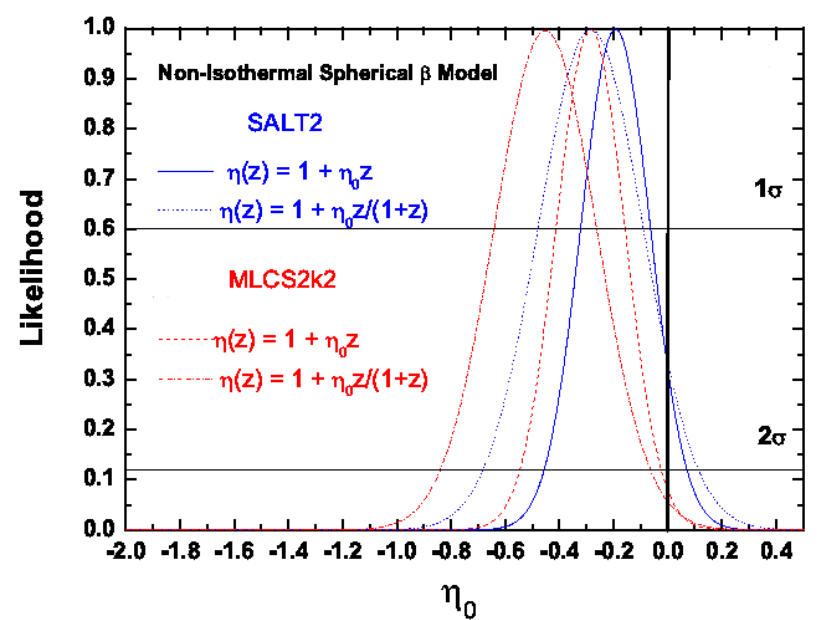

Fig. 8. (Color online) a) Likelihood distribution functions for the Bonamente et al. (2006) sample for both parametrizations and both $\mathrm{SNe}$ Ia light-curve fitters.

compared to distances from the elliptical model and the $\Lambda \mathrm{CDM}$ model (WMAP7). For the Bonamente et al. (2006) sample the DD relation validity is marginally compatible $(2 \sigma)$ with SALT2 and at $3 \sigma$ with MLCS2K2.

\section{Searching for the true geometry of clusters: a joint analysis}

In the previous sections we have discussed how the validity of the DD relation can be used to fix the true cluster geometry by using two independent approaches: (i) the model-dependent test based on the SZE/X-ray technique in the context of the $\Lambda$ CDM model (WMAP7 analysis), and (ii) the model-independent test using the combination of SZE/X-ray for galaxy clusters and SNe Ia data. For the former case the elliptical description provides the best fit supporting the standard DD relation in $\Lambda$ CDM (Komatsu et al. 2011). On the other hand, the isothermal spherical $\beta$ model is more compatible with the validity of the DD relation in the model-independent test involving supernovas. Since likelihoods for both descriptions in both approaches (see Figs. 2a and 5a) are compatible with each other in at least 2-sigma, it is convenient to perform a joint analysis involving these independent and complementary treatments in order to choose the better description.

Naturally, one may argue that the joint analysis will be plagued by the underlying tension between $\mathrm{SNe}$ and $\mathrm{CMB}$ data. In this context, we recall that this tension was recently discussed by taking into account weak-lensing effects caused by inhomogeneities at low and intermediate redshifts by Amendola et al. (2010). By using the Union data (2008), they showed that the inclusion of lensing moves the best-fit model significantly toward the flat LCDM model (owing to corrections induced by the lens convergence on the distance modulus). This treatment is beyond the scope of our paper. Nevertheless, this treatment is valid in the same sense as the early analysis involving SNe Ia and CMB were to the cosmic concordance model. As we shall see, the joint analysis below confirms that the elliptical isothermal model provides the best description for galaxy clusters.

Now, by adding the $\chi^{2}$ statistics for the different approaches, the resulting likelihoods for the $\eta_{0}$ parameter were again obtained. The main findings for both parametrizations (linear and non-linear cases) are summarized in Table 4 . Note that we 
Table 4. The $\eta_{0}$ values for our joint analysis. All errors are at $1 \sigma$ and the $\chi^{2} /$ d.o.f. is given in parentheses.

\begin{tabular}{lccc}
\hline \hline Isothermal elliptical $\beta$ model & $\begin{array}{c}\text { WMAP+SNe Ia } \\
\text { Constitution }\end{array}$ & $\begin{array}{c}\text { WMAP+SNe Ia } \\
\text { SDSS(SALT2) }\end{array}$ & $\begin{array}{c}\text { WMAP+SNe Ia } \\
\text { SDSS(MLCs2k2) }\end{array}$ \\
\hline$\eta_{0}$ (Linear case) & $-0.096_{-0.084}^{+0.096}(0.96)$ & $-0.08_{-0.09}^{+0.09}(0.93)$ & $-0.11_{-0.12}^{+0.10}(0.97)$ \\
$\eta_{0}$ (Non-linear case) & $-0.14_{-0.13}^{+0.13}(0.97)$ & $-0.12_{-0.13}^{+0.12}(0.95)$ & $-0.177_{-0.127}^{+0.143}(0.96)$ \\
Isothermal spherical $\beta$ model & WMAP + SNe Ia & WMAP+SNe Ia & WMAP+SNe Ia \\
& Constitution & SDSS $($ SALT2) & SDSS(MLCS2k2) \\
$\eta_{0}$ (Linear case) & $0.178_{-0.108}^{+0.097}(0.76)$ & $0.195_{-0.115}^{+0.105}(0.77)$ & $0.16_{-0.11}^{+0.12}(0.75)$ \\
$\eta_{0}$ (Non-linear case) & $0.263_{-0.148}^{+0.117}(0.75)$ & $0.29_{-0.15}^{+0.14}(0.76)$ & $0.24_{-0.148}^{+0.152}(0.75)$ \\
\hline
\end{tabular}

restricted our attention to the De Filippis et al. (2005) samples (elliptical and spherical isothermal assumptions).

From Table 4, we see that the elliptical geometry agrees better with the strict validity of the duality relation when both approaches are considered. This result remains valid regardless of the supernova sample (Constitution or SDSS) adopted, the parameterization (linear or non-linear), or the kind of light fitter used.

In Fig. 9, we plotted the likelihoods function for the $\eta_{0}$ parameter as obtained from our joint analysis by considering again the De Filippis et al. (2005) samples (elliptical and spherical isothermal geometries). Figure $9 \mathrm{a}$ is the joint analysis for the WMAP + SNe Ia by using the Constitution sample while in Figs. $9 \mathrm{~b}$ and $\mathrm{c}$, we considered SDSS sample, but now taking into account the different light-curve fitters (SALT2 and MLCS2k2). The horizontal lines are cuts in the regions of $68.3(1 \sigma)$ per cent and $95.4(2 \sigma)$ per cent probability. Our joint analysis shows that an elliptical geometry is suggested by the existing data even when we take into account the SNe Ia light-curve fitters. Because several SZE surveys are in progress, our results call attention to a basic difficulty involving the spherical assumption to describe the cluster morphology when independent cosmological probes are considered.

\section{Conclusions}

We explored some physical consequences of a deformed distance-duality relation, $\eta(z)=D_{\mathrm{L}}(1+z)^{-2} / D_{\mathrm{A}}$, based on observations of galaxy clusters using SZE and X-ray surface brightness. The $\eta(z)$ parameter was described by two distinct forms, $\eta=1+\eta_{0} z$ and $\eta=1+\eta_{0} z /(1+z)$, thereby recovering the standard equality between distances for extremely low redshifts.

Initially, we discussed the consistency between the strict validity of the distance-duality relation and the underlying assumptions about the galaxy cluster geometries. In our analysis we used angular diameter distances from galaxy clusters of the De Filippis et al. (2005) and Bonamente et al. (2006) samples. The former sample consists of 25 data sets where the galaxy clusters were described by an isothermal elliptical $\beta$ model and a spherical $\beta$ model, whereas the latter sample consists of 38 data sets where a non-isothermal spherical double $\beta$ model was used. We showed that the elliptical geometry is more consistent with no violation of the distance duality relation in the context of $\Lambda$ CDM (WMAP7 data). In the elliptical case of the De Filippis et al. (2005) sample (see Fig. 2a and Table 1) we found $\eta_{0}=-0.056_{-0.1}^{+0.1}$ and $\eta_{0}=-0.088_{-0.14}^{+0.14}$ for linear and nonlinear parametrizations at $1 \sigma$ (statistical plus systematic errors), respectively. The analysis with the isothermal spherical $\beta$ model led to higher and positive $\eta_{0}$ values than those from elliptical $\beta$ model, indicating that the estimated angular distances with the spherical $\beta$ model are overestimated compared to those from
$\Lambda \mathrm{CDM}$ (WMAP7). On the other hand, the non-isothermal spherical double $\beta$ model (see Fig. 2b) was only marginally compatible with $\eta_{0}=0$, such as $\eta_{0}=-0.12_{-0.12}^{+0.12}$ and $\eta_{0}=-0.175_{-0.175}^{+0.175}$ for linear and non-linear parameterizations at $2 \sigma$ (statistical plus systematic errors), respectively. In this way, our analysis revealed that the elliptical model is compatible with the duality relation at $1 \sigma$, whereas the non-isothermal spherical model (in Bonamente et al. 2006 sample) is only marginally compatible at $3 \sigma$ in the $\Lambda$ CDM framework.

Furthermore, we discussed a new and model-independent cosmological test for the distance-duality relation. We considered the three angular diameter distance samples from galaxy clusters, which are obtained by using SZE and X-ray surface brightness, together the luminosity distances given by two subsamples of SNe Ia taken from the Constitution data. For each subsample, the redshifts of the SNe Ia were carefully chosen to coincide with those of the associated galaxy cluster sample $(\Delta z<0.005)$. Our results showed that the confrontation between the angular diameter distances from the De Filippis et al. (2005) sample (elliptical model) with $\mathrm{SNe}$ Ia data pointed to a moderate violation of the reciprocity relation (the DD relation was marginally satisfied in $2 \sigma$ ) and the $\eta_{0}$ values were predominantly negative. Since $(1+z)^{2} D_{\mathrm{A}}^{\text {data }}(z) / D_{\mathrm{L}}(z) \propto 1+\eta_{0} z$, negative $\eta_{0}$ values indicated luminosity distances overestimated with relation to angular diameter ones. This tension between Sne Ia and the elliptical De Filippis et al. (2005) sample arises because SNe Ia samples prefer universes with higher $\Omega_{\Lambda}$ values than the WMAP7 results. When an isothermal spherical $\beta$ model is adopted, the DD relation had been satisfied at $1 \sigma$ with $\eta_{0}$ values preferably positive. Seemingly, this concordance occurred only because an isothermal spherical $\beta$ model yields angular distances overestimated in comparison to an elliptical model and WMAP7 results. For the Bonamente et al. (2006) sample, where a non-isotermal spherical double $\beta$ model was assumed to describe the clusters, we saw that the DD relation is not obeyed even at $3 \sigma$. For this case we obtained $\eta_{0}=-0.42_{-0.34}^{+0.34}$ and $\eta_{0}=-0.66_{-0.5}^{+0.5}$ for linear and non-linear $\eta(z)$ parameterizations in $3 \sigma$ (statistical plus systematic errors), respectively.

Moreover, by using the SNe Ia data from SDSS-II (Kessler et al. 2009), we explored the influence of the two different SNe Ia light-curve fitters in our model-independent cosmological test for the DD relation. In this way, we considered two subsamples of SNe Ia taken from SDSS-II (2007) for $D_{\mathrm{L}}$ where, again, the $\mathrm{SNe}$ Ia redshifts of each subsample were carefully chosen to coincide with those of the associated galaxy cluster sample $(\Delta z<0.005)$. In this case, each subsample of SNe Ia was analyzed by the MLCS2K2 and SALT2 light-curve fitters separately. Interestingly, we obtained conflicting results between the two light-curve fitters. For both light-curve fitter-method the best fits are obtain in an analysis involving the elliptical De Filippis et al. (2005) sample. In this case, the DD relation validity was 
R. F. L. Holanda et al.: Probing cosmic distance duality relation

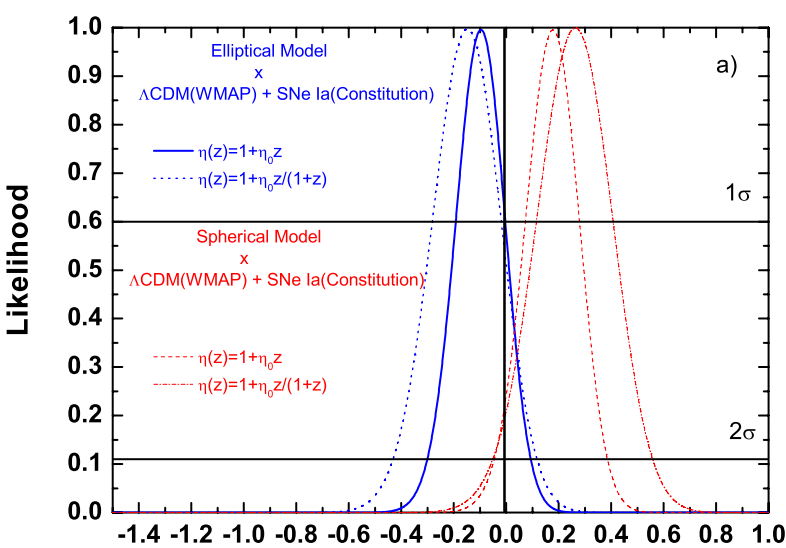

$\eta_{0}$

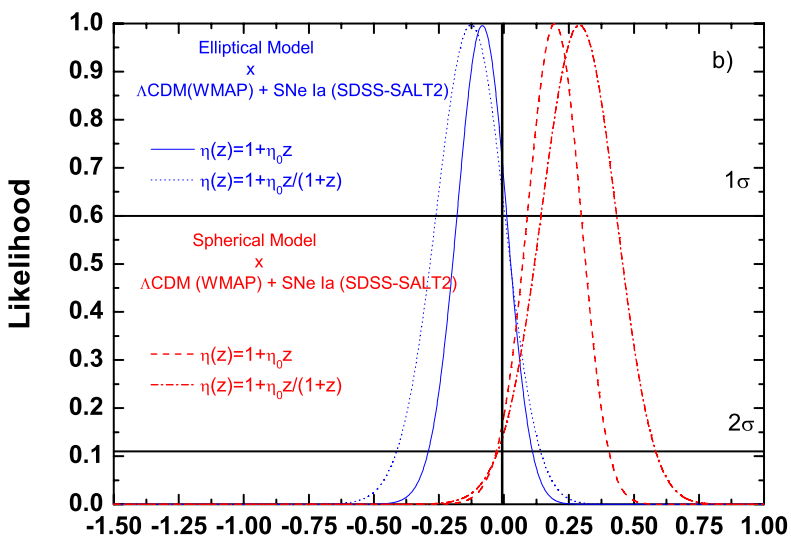

$\eta_{0}$

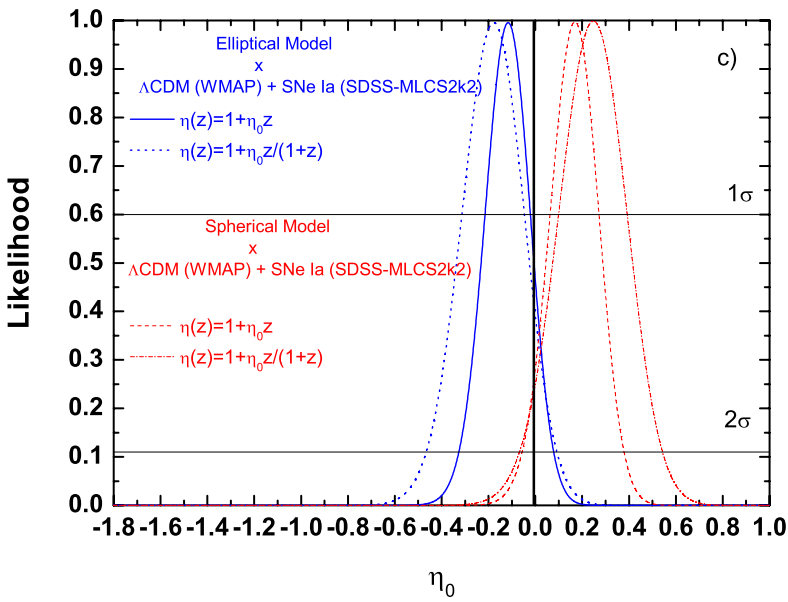

Fig. 9. (Color online) a) Joint analysis for the De Filippis et al. (2005) samples and WMAP + SNe Ia (Constitution Sample). b) Joint analysis for the De Filippis et al. (2005) samples and WMAP + SNe Ia (SDSSSALT2). c) Joint analysis for the De Filippis et al. (2005) samples and WMAP + SNe Ia (SDSS-MLCS2k2).

obtained in $1 \sigma$ with the SALT2 method and it was marginally compatible with MLC2K2 method with $\eta_{0}$ values considerably negative. This result points to overestimated luminosity distances when SNe Ia are analyzed with MLC2K2 method. For this light-curve fitter, the isothermal spherical $\beta$ model provided that the DD relation was satisfied at $1 \sigma$. For all tests of the Bonamente et al. (2006) sample, the DD relation validity is obtained marginally at $2 \sigma$ (SALT2) and at $3 \sigma$ (MLCS2K2). We stress that our results are independent of the redshifts chosen to realize the test (of the galaxy clusters or SNe Ia), thereby showing the robustness of the method and supporting $\Delta z<0.005$ as a fair choice.

Finally, we applied a joint analysis involving these independent and complementary treatments by adding the $\chi^{2}$ statistics for the different approaches. Interestingly, we saw that the elliptical geometry agrees better with the strict validity of the duality relation when both approaches were considered. This result remains valid regardless of the adopted $\mathrm{SNe}$ Ia sample (Constitution or SDSS), the parameterization (linear or nonlinear), or the kind of light-curve fitter used (see Table 4). The non-isothermal spherical double $\beta$ model was only marginally compatible with the DD relation validity in all treatments.

Summarizing, the statistical analysis presented here provides new evidence that the true geometry of clusters has an elliptical form. In principle, it is remarkable that a local property such as the geometry of galaxy clusters may be constrained by a global argument like the one provided by the cosmological distanceduality relation. This result also reinforces the interest in the observational search for SZE and X-ray from clusters at high redshifts. In the near future, when more and larger samples with smaller statistical and systematic uncertainties become available, the method proposed here, based on the validity of the distanceduality relation, can improve the limits on the possible cluster geometries and explore systematic errors in $\mathrm{SNe}$ Ia and galaxy clusters observations.

Acknowledgements. R.F.L.H. is supported by FAPESP (No. 07/52912-2) and J.A.S.L. is partially supported by CNPq (No. 306054/2010) and FAPESP (No. 04/13668-0).

\section{References}

Albani, V. V. L., Iribarrem, A. S., Ribeiro, M. B., \& Stoeger, W. R. 2007, ApJ, 657,760

Amanullah, R., Lidman, C., Rubin, D., et al. 2010, ApJ, 716, 712

Astier, P., Guy, J., Regnault, N., et al. 2006, A\&A, 447, 31

Avgoustidis, A., Burrage, C., Redondo, J., Verde, L., \& Jimenez, R. 2010, JCAP, 10,024

Basset, B. A., \& Kunz, M. 2004, Phys. Rev. D, 69, 101305

Bengochea, G. R. 2011, PLB, 696, 5

Bonamente, M., Joy, M. K., Carlstrom, J. E., Reese, E. D., \& LaRoque, S. J. 2004, ApJ, 614, 56

Bonamente, M., Joy, M. K., LaRoque, S. J., et al. 2006, ApJ, 647, 25

Cavaliere, A., \& Fusco-Fermiano, R. 1978, A\&A, 667, 70

Cunha, J. V., Marassi, L., \& Santos, R. C. 2007a, IJMPD, 16, 403

Cunha, J. V., Marassi, L., \& Lima, J. A. S. 2007b, MNRAS, 379, L1

Daly, R. A., \& Djorgovski, S. G. 2003, ApJ, 597, 9

Davis, T. M., Mörtsell, E., Sollerman, J., et al. 2007, ApJ, 666, 716

De Bernardis, F., Giusarma, E., \& Melchiorri, A. 2006, IJMPD, 15, 759

D’Agostini, G. 2004, unpublished [physics/0403086]

De Filippis, E., Sereno, M., Bautz, M. W., \& Longo, G. 2005, ApJ, 625, 108

Ebeling, H., Voges, W., Bohringer, H., et al. 1996, MNRAS, 281, 799

Ellis G. F. R. 1971, Relativistic Cosmology, Proc. Int. School Phys. Enrico Fermi, ed. R. K. Sachs (New York: Academic Press), 104; reprinted in Gen. Rel. Grav., 41, 581, 2009

Ellis, G. F. R. 2007, Gen. Rel. Grav., 39, 1047

Etherington, I. M. H. 1933, Phil. Mag., 15, 761; reprinted in Gen. Rel. Grav., 39, 1055,2007

Gonçalves, R. S., Holanda, R. F. L., \& Alcaniz, J. S. 2012, MNRAS, 420, L43

Gurvitz, L. I. 1994, ApJ, 425, 442

Gurvitz, L. I., Kellerman, K. I., \& Frey, S. 1999, A\&A, 342, 378

Guy, J., Astier, P., Baumont, S., et al. 2007, A\&A, 466, 11

Jha, S., Riess, A. G., \& Kirshner, R. P. 2007, ApJ, 659, 122

Hicken, M., Wood-Vasey, W. M., Blondin, S., et al. 2009, ApJ, 700, 1097

Holanda, R. F. L., Lima, J. A. S., \& Ribeiro, M. B. 2010, ApJ, 722, L233

Holanda, R. F. L., Lima, J. A. S., \& Ribeiro, M. B. 2011, A\&A, 528, L14

Itoh, N., Kohyama, Y., \& Nozawa, S. 1998, ApJ, 502, 7

Kessler, R., Becker, A. C., Cinabro, D., et al. 2009, ApJ, 185, 32

Komatsu, E., Smith, K. M., Dunkley, J., et al. (WMAP collaboration) 2011 , ApJS, 192, 18

Kowalski, M., Ackermann, M., Adams, J., et al. 2008, ApJ, 749, 686 
A\&A 538, A131 (2012)

La Roque, S. J., Bonamente, M., Carlstrom, J. E., et al. 2006, 652, 917 Li, Z., Wu, P., \& Yu, H. 2011, 729, L14

Liang, N., Cao, S., \& Zhu, Z. 2011 [arXiv: 1104.2497v1]

Lima, J. A. S., \& Alcaniz, J. S. 2000, A\&A, 357, 393

Lima, J. A. S., \& Alcaniz, J. S. 2002, ApJ, 566, 15

Lima, J. A. S., Cunha, J. V., \& Alcaniz, J. S. 2003, Phys. Rev. D, 68, 023510

Lima, J. A. S., Cunha, J. V., \& Zanchin, V. T. 2011, ApJ, 742, L26

Linder, E. V. 2003, PRL, 90, 091301

Mantz, A., Allen, S. W., Rapetti, D., \& Ebeling, H. 2010, MNRAS, 406, 1759

Mason, B. S., Myers, S. T., \& Readhead, A. C. S. 2001, ApJ, 555, L11

Meng, X.-L., Zhang, T.-J., \& Zhan, H. 2012, ApJ, 745, 98

Mohr, J. J., Mathiesen, B., \& Evrard, A. E. 1999, ApJ, 517, 627

Morandi, A., Pedersen, K., \& Limousin, M. 2010, ApJ, 713, 491

Nair, R., Jhingan, S., \& Jain, D. 2011, JCAP, 05, 023

Navarro, J. F., Frenk, C. S., \& White, S. D. M. 1997, 490, 493

Nozawa, S., Itoh, N., \& Kohyama, Y. 1998, ApJ, 508, 17

Padmanabhan, T., \& Choudury, R. 2003, MNRAS, 344, 823

Percival, W., Reid, B. A., Eisenstein, D. J., et al. 2010, MNRAS, 401, 2148

Rangel Lemos, L. J., \& Ribeiro, M. B. 2008, A\&A, 488, 55

Reese, E. D. 2004, in Measuring and Modeling the Universe, ed. W. L. Freedman (CUP), 138

Reese, E. D., Carlstrom, J. E., Joy, M., et al. 2002, ApJ, 581, 53
Reiprich, T. H., \& Bohringer, H. 2002, ApJ, 567, 716

Ribeiro, M. B. 1992, ApJ, 388, 1

Ribeiro, M. B. 1993, ApJ, 415, 469

Ribeiro, M. B. 2005, A\&A, 429, 65

Ribeiro, M. B., \& Stoeger, W.R. 2003, ApJ, 592, 1

Riess, A., Macri, L., Casertano, S., et al. 2009, ApJ, 116, 1009

Santos, R. C., \& Lima, J. A. S. 2008, Phys. Rev. D, 77, 083505

Sarazin, C. L. 1988, in X-ray emission from clusters of galaxies, Cambridge Astrophysics Series (Cambridge University Press)

Schneider, P., Ehlers, J., \& Falco, E. E., 1992, Gravitational Lenses (Berlin: Springer-Verlag)

Shang, C., Haiman, Z., \& Verde, L. 2009, MNRAS, 400, 2, 1085

Silva, R., Alcaniz, J. S., \& Lima, J. A. S. 2007, IJMPD, 16, 469

Sollerman, J., Mörtsell, E., Davis, T. M., et al. 2009, ApJ, 703, 1374

Spergel, D. N., Verde, L., Peiris, H. V., et al. 2003, ApJS, 148, 175

Stern, D., Jimenez, R., Verde, L., Kamionkowski, M., \& Stanford, S. A. 2010, JCAP, 02, 008

Sunyaev, R. A., \& Zel'dovich, Ya. B. 1972, Comments Astrophys. Space Phys., 4, 173

Uzan, J. P., Aghanim, N., \& Mellier, Y. 2004, Phys. Rev. D, 70, 083533

Wang, J., \& White, S. D. M. 2009, MNRAS, 396, 709

Wei, H. 2010, PLB, 687, 286 